\title{
Experimental investigation of higher harmonic wave loads and moments on a vertical cylinder by a phase-manipulation method
}

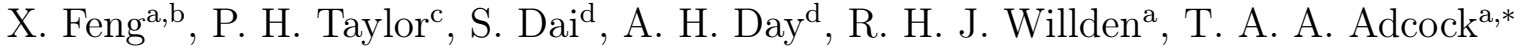 \\ ${ }^{a}$ Department of Engineering Science, University of Oxford, OX1 3PJ, UK \\ ${ }^{b}$ Department of Ocean Science and Engineering, Southern University of Science and Technology, Shenzhen, \\ 518055, China \\ ${ }^{c}$ Faculty of Engineering and Mathematical Sciences, University of Western Australia, WA6009, Australia \\ ${ }^{d}$ Department of Naval Architecture, Ocean and Marine Engineering, University of Strathclyde, G4 OLZ, UK
}

\begin{abstract}
This paper considers wave loads on bottom mounted vertical cylinders in the regime relevant to offshore wind turbine monopiles, examining the higher harmonics of the loading from nonbreaking waves which are important in engineering design. Although we consider measurements of total force, our focus is on the moments applied about the seabed which have received much less attention in the literature. We consider an empirical 'Stokes-like' model whereby the magnitude of the higher harmonics of force or moment are dependent on the linear force or moment timeseries raised to an appropriate power and a fixed coefficient. We compare this representation to high quality wave flume measurements and fully non-linear potential flow simulations. In general we find good agreement for all of the harmonic structure except for the triple frequency component where there are significant discrepancies. We also extend the Stokes-like model and show that harmonics of moments can be robustly predicted from the force harmonic and an appropriate constant moment arm for each harmonic, so a more elaborate calculation is generally not necessary.
\end{abstract}

Keywords: Nonlinear diffraction, focused wave groups, higher harmonics, wave loads

\section{Introduction}

In many locations around the world ocean waves produce important dynamic loads on offshore wind turbines. The typical relatively small Keulegan-Carpenter $(K C)$ number for offshore wind turbine monopiles implies that inertia loading will dominate and drag forces can be neglected 5 for non-breaking waves. The inertia load produces a force at the same frequencies as the incom-

\footnotetext{
${ }^{*}$ Corresponding author

Email address: thomas.adcock@eng.ox.ac.uk (T. A. A. Adcock)
} 
ing wave. However, there will be higher harmonics in the loading from large waves and these can make up a significant part of the magnitude of the total load. The higher harmonic loads will tend to act near the free surface and therefore have a higher moment arm than the linear loads - and hence increase moment around the sea-bed. However, perhaps most importantly these higher harmonic loads may potentially act at around the lowest natural frequency for which an offshore wind turbine is designed for (see Kallehave et al. 2015). The resonance associated with column-supported offshore structures has been described as 'ringing'. The higher-harmonic ringing loads on a vertical surface-piecing cylinder have been investigated in the literature e.g. (Krokstad and Stansberg, 1995; Stansberg, 1997; Chaplin et al., 1997; Grue and Huseby, 2002; and geotechnical design and therefore improving the understanding of the higher harmonics of wave loading is of importance. Further, the approach in the present paper moves towards developing computationally fast methods of including these components in time-domain simulations of structural and geotechnical responses where complex fluid mechanics simulations or experiments are not practical.

Analytical approaches have been developed for calculating higher harmonic loads on cylinders. Second-order diffraction theory has been developed by a number of authors (Kim and Yue, 1989; Chau and Eatock Taylor, 1992; Huang and Eatock Taylor, 1996; Newman, 1996). This was pushed further by Faltinsen et al. (1995). They developed a third-order diffraction model (referred to as FNV) for approximating the third-order wave force on a slender cylinder, based on the assumptions of deep water and that the incident wave amplitude is in the same order as the cylinder radius. Recent experiments (Huseby and Grue, 2000; Chen et al., 2018; Kristiansen and Faltinsen, 2017) have generally shown good agreement with FNV theory in the regime for which the theory is valid, although some discrepancy in phase is apparent. Malenica and Molin (1994, 1995) (referred as M\&M) made a complete third-order diffraction analysis following the perturbation method and utilising a semi-analytical solution for the third-order potential. The predictions of FNV model and M\&M differ appreciably in the magnitude of the third-order force when the incident frequency became relatively high and markedly in the phase of the force. As the underlying perturbation assumptions in FNV and M\&M are different, the results of one cannot be regarded as a special case of the other. Teng and Kato (2002) also reported the third-order wave loading at the triple frequency on fixed truncated bodies. Good agreements between their model and experiments were found at low wave frequencies; however, their results at third order 
were under-predicted at high wave frequencies. For harmonics higher than the third there are at present no analytical solutions. However, Chen et al. (2018) have shown experimentally that the loading above this can be modelled using a harmonic type representation. The recent numerical work in Chen et al. (2019) shows that higher harmonic loads can be extracted even considering the interactions of waves and sheared currents.

In the regular wave tests in Kristiansen and Faltinsen (2017), discrepancies between the FNV model and measurements at the third harmonic were found to increase with wave steepness. The

The experimental and numerical data sets are complementary rather than the primary purpose being for one to directly validate the other although where there are agreements and discrepancies is obviously of interest. There are subtle differences between the exact scenarios due to the nature 
of the tests. Most obviously, the numerical simulations are limited in the maximum steepness that can be run due to the nature of the code, whereas the experiments are limited by the lowest steepness that can be run due to measurement accuracy of the small higher harmonics. Other differences include the column not extending to the bed in the experiment (for practical reasons) although it does in the numerics.

\section{Methods}

\subsection{Experimental setup}

The experimental campaign was undertaken at the Kelvin Hydrodynamics Laboratory at the University of Strathclyde, Glasgow, United Kingdom. The tests were carried out in the lab's 76 $\mathrm{m}$ long, $4.6 \mathrm{~m}$ wide wave flume with a constant water depth of $1.8 \mathrm{~m}$. The tank is equipped with a hinged 'flap-type' wavemaker at one end consisting of four paddles with force-feedback wave absorption. The four paddles are side-by-side extended over the width of the tank, and they are prescribed with the same input wave signal - we generate two-dimensional waves. A 13 m-long sloping beach covered with a porous carpet acts as a passive absorber at the other end. A single surface-piercing vertical cylinder of diameter $0.315 \mathrm{~m}$ was placed at the centreline of the tank, with its centre $35.315 \mathrm{~m}$ away from the wavemaker. Figure 1 shows a sketch of the experimental setup. As the tank width is not large compared with the peak wavelength we tested, reflection from the side walls is expected. We select the appropriate time window such that the wave profile at the wave focusing is not disturbed by the reflected waves from the side walls. Additionally, this possible reflection will not significantly affect much the inline wave forces due to symmetry and these reflected waves travelling normal to the inline direction.

The cylinder was rigidly supported at its upper end by a six-degree-of-freedom load cell mounted on a stiff frame (see Figure 2(a)). The bottom of the cylinder was fixed to a frame attached to the tank floor via a three-degree-of-freedom load cell attached to a bearing assembly which released the bending moments. The lower support raised the end of the cylinder slightly above the tank floor; the resulting cylinder draught was $1.6 \mathrm{~m}$. The upper end of the cylinder is located just under $1.0 \mathrm{~m}$ above the still water surface, to allow for the substantial run-up of the large focused waves. The arrangement allows the calculation of the total inline force and the inline moment about the bearing, so that the moment arm may be found. Figure 2(b) shows a wide view of the cylinder viewed from the wavemaker as a wave passes, with the inset image showing the side view. The setup was found to have a relatively high system natural frequency of 


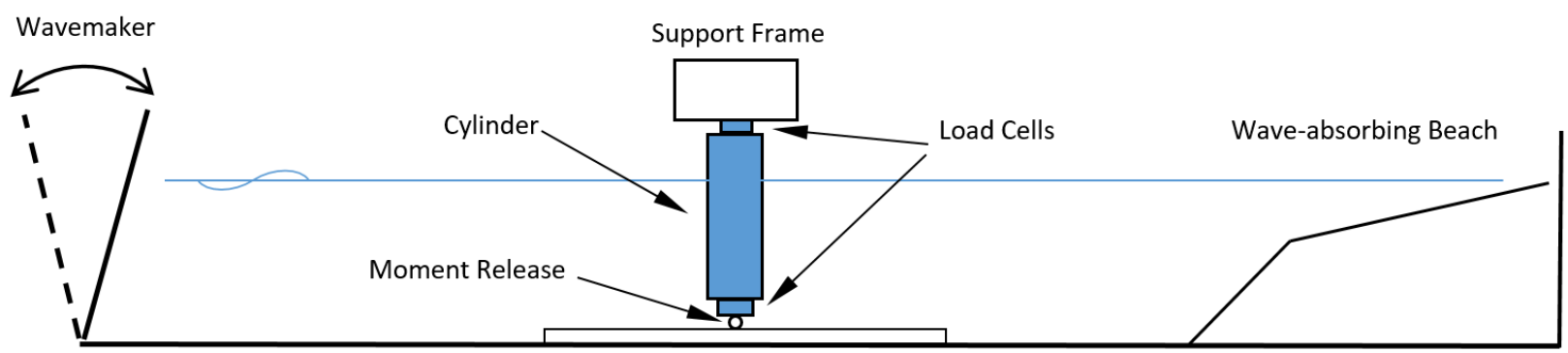

Figure 1: The schematic overview of the experimental setup.

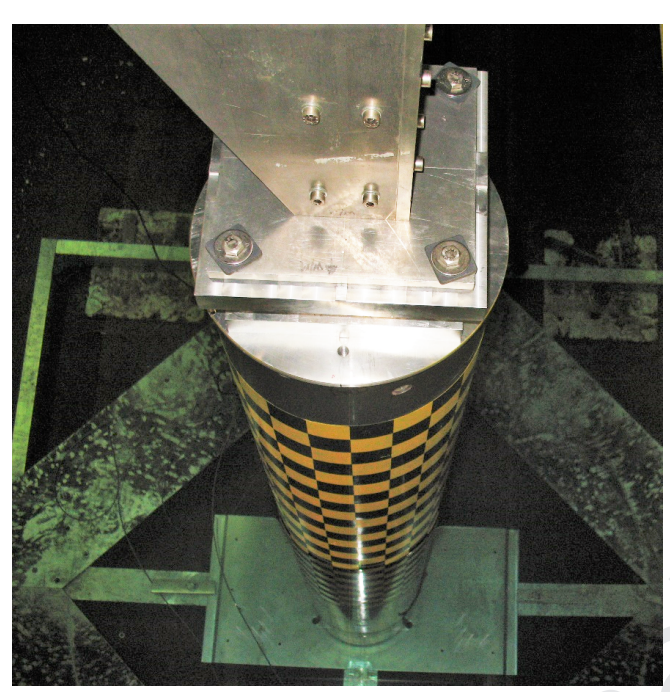

(a)

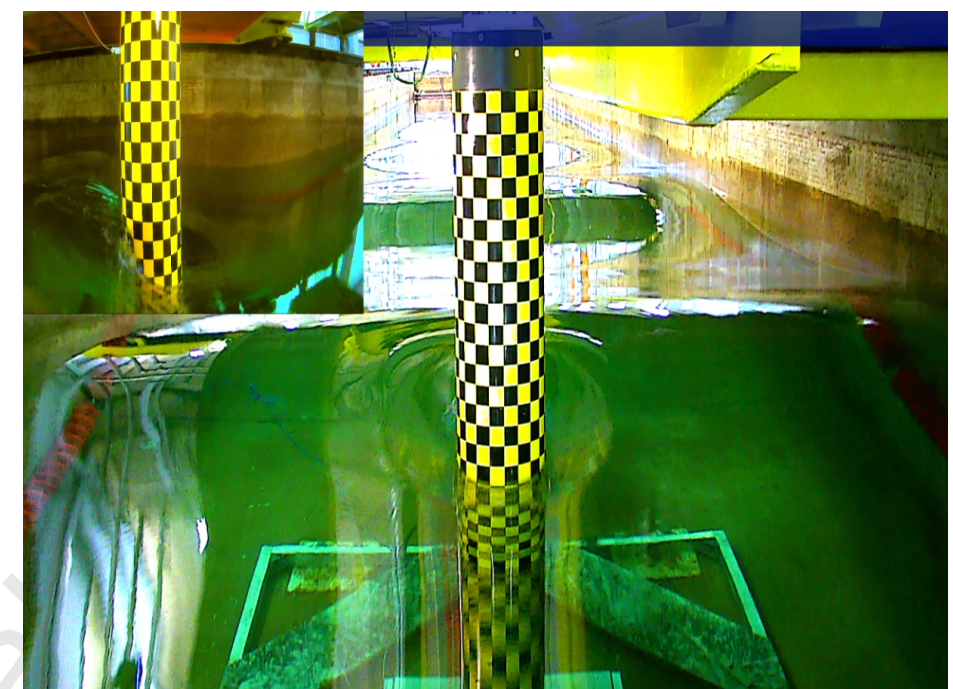

(b)

Figure 2: The experimental setup in the Kelvin Hydrodynamic Laboratory (a) load cell on top of the cylinder; (b) the vertical cylinder in the tank. a high natural frequency suggests the possible cylinder dynamics is negligible in the frequency range of interest, allowing the measurement of the pure hydrodynamic loads without the necessity of eliminating the contribution due to structural dynamics (see Chen et al. 2018).

In this study we use wave groups to load the structure. These have the advantage over regular or random waves that there are no issues with reflections either from the beach or the side of the tank. We use the NewWave approach (see Tromans et al. 1991) so that the wave that would hit the structure would be the expected shape of an extreme event in an input seas state. As wave-group evolution has significant non-linearities in unidirectional waves (Lo and Mei, 1985; Baldock et al., 1996; Adcock and Taylor, 2009) iteration was used to produce the focussed wave group. The input timeseries used is identical to that used in the study of Santo et al. (2018).

A JONSWAP spectrum of peak frequency $f_{p}$ with $\gamma=3.3$ was used to generate the wave 


\begin{tabular}{cccccccc}
\hline Case & $f_{p}(\mathrm{~Hz})$ & $k_{p}\left(m^{-1}\right)$ & $k_{p} R$ & $k_{p} h$ & $A(m)$ & $k_{p} A$ & $K C$ \\
\hline 1 & 0.429 & 0.82 & 0.129 & 1.476 & 0.134 & 0.110 & 2.671 \\
2 & 0.429 & 0.82 & 0.129 & 1.476 & 0.147 & 0.120 & 2.927 \\
3 & 0.429 & 0.82 & 0.129 & 1.476 & 0.160 & 0.131 & 3.185 \\
4 & 0.429 & 0.82 & 0.129 & 1.476 & 0.169 & 0.138 & 3.366 \\
5 & 0.429 & 0.82 & 0.129 & 1.476 & 0.179 & 0.146 & 3.562 \\
6 & 0.429 & 0.82 & 0.129 & 1.476 & 0.206 & 0.169 & 4.110 \\
7 & 0.429 & 0.82 & 0.129 & 1.476 & 0.241 & 0.197 & 4.802 \\
8 & 0.429 & 0.82 & 0.129 & 1.476 & 0.256 & 0.210 & 5.115 \\
9 & 0.477 & 0.973 & 0.153 & 1.751 & 0.162 & 0.158 & 3.237 \\
10 & 0.531 & 1.169 & 0.184 & 2.104 & 0.160 & 0.188 & 3.200 \\
\hline
\end{tabular}

Table 1: Test parameters. The peak frequency $f_{p}$ is used in JONSWAP spectrum. The wavenumber $k_{p}$ for the wave group is computed from the linear dispersion relation using the peak frequency. Amplitude $A(m)$ is the linear component of the focused wave group defined in the NewWave model. The Keulegan-Carpenter number $(K C)$ is computed by $K C=\pi A / R$, where $R$ is the cylinder radius and $A$ is the target amplitude.

groups. The discretized spectrum cut-off is $0.5 f_{p}-3.0 f_{p}$. Wave groups with varying wave steepness were tested to investigate the nonlinear effects on the higher-order wave loads. The tested wave groups are listed in Table 1.

The test conditions give a range of the ratio $A / R=(0.6-1.9)$, that is, the wave group amplitude is mostly of the same order with the cylinder radius, $R$ (implying the FNV model for third-order force ought to be applicable). The corresponding wave steepness $k_{p} A$ is $(0.10-0.25)$. No wave breaking of the undisturbed waves is expected or was observed in this range. With the $K C$ number smaller than 5 (except Case 8), we expect the wave force to be inertia dominated. Local viscous effects near focusing might be expected to play a role when $K C>4.0$. However, we see little evidence of this in the present paper and viscous effects appear relatively unimportant in this analysis. In particular, we do not think these explain the discrepancy between the potential flow model and experimental results in a few cases.

\subsection{Numerical model}

The fully nonlinear potential flow model 'OxPOT' presented in Bai and Eatock Taylor (2006) and Bai et al. (2014) is employed. The model solves the velocity potential satisfying the Laplace equation in the time domain. The exact free-surface dynamic and kinematic boundary conditions are satisfied. A rectangular Numerical Wave Tank is set up in the model where a piston-type wavemaker is located at one end of the tank and a numerical damping beach at the other. Impermeable conditions are satisfied on the tank side walls and bottom and on the fixed structure wetted surface. Waves are generated and controlled through the movement of the wavemaker 
which is modelled as a horizontally moving solid wall. The boundary value problem for the fluid flow enclosed by the tank, and the body wetted surface and the free water surface is solved numerically in the time domain.

A higher-order boundary element method is employed to formulate the boundary integral equations (BIEs). The computational boundaries are discretized by quadratic isoparametric elements. The quantities within an element can be computed through its nodal points using local shape functions. For discretization, structured 8-node quadrilateral elements are distributed on the vertical tank walls and body surfaces. On the free surface, unstructured 6- node triangular elements are generated by Delaunay triangulation. Mesh density is controlled by the mesh size at the edges of the boundaries. Once the velocity potential and velocity are obtained by solving a set of algebraic linear equations resulting from BIEs, the hydrodynamic forces acting on the structures can be computed by integrating the pressure over the structure surfaces.

During the simulation, the exact free surface is captured at every time step. To update the nonlinear free surface in the time domain, the Mixed Eulerian-Lagrangian algorithm is adopted in the model. The free surface nodes follow the Lagrangian trajectory of the fluid particles and are interpolated to the new surface in the Eulerian frame. The time marching scheme is the 4th-order Runge-Kutta algorithm, which has been shown to be numerically stable and accurate. Detailed numerical implementation on the numerical wave tank techniques, such as the damping beach and numerical integration with a free-surface/solid boundary singularity, can be found in Bai and Eatock Taylor (2006) and Bai et al. (2014).

In the numerical simulation, the cylinder is modelled as bottom mounted, i.e. its draft is 1.8 $\mathrm{m}$. The numerical tank is shorter than the experimental to save on computation time. The length is $35 \mathrm{~m}$ long and the width remains the same as the physical tank. This length is about 4 times the dominant wavelength and is long enough to ensure the water surface near the cylinder is free from reflection. The numerical damping beach at the end of the tank is shown to be effective in the previous studies Bai et al. (2014) and Feng et al. (2019). The same JONSWAP spectrum is used to define the incident wave group in both the simulations and experiments.

\subsection{Harmonic decomposition}

In this paper we utilise phase controlled harmonic decomposition to extract the different harmonics. With the assumption of a relatively narrow-banded spectrum, the focused wave group can be assumed to have a slowly-varying amplitude $A(t)$ near focusing and the wave force 
is expanded with respect to wave steepness as

$$
\begin{aligned}
F(t) & =\Re\left[A \mathcal{F}_{11} \exp (ß \omega t)+A^{2}\left(\mathcal{F}_{20}+\mathcal{F}_{22} \exp (2 ß \omega t)\right)+A^{3}\left(\mathcal{F}_{31} \exp (ß \omega t)+\mathcal{F}_{33} \exp (3 ß \omega t)\right)\right. \\
& \left.+A^{4}\left(\mathcal{F}_{40}+\mathcal{F}_{42} \exp (2 ß \omega t)+\mathcal{F}_{44} \exp (4 ß \omega t)\right)+O\left(A^{5}\right)\right]
\end{aligned}
$$

up to fourth order of the steepness $k A$. The coefficients $\mathcal{F}_{m n}$ represent complex functions corfour runs are carried out with phases: $\varphi_{0}=0^{\circ}, 90^{\circ}, 180^{\circ}$ and $270^{\circ}$. By linearly combining the four corresponding responses $F_{0}, F_{90}, F_{180}$ and $F_{270}$, harmonics may be separated:

$$
\begin{aligned}
\left(A \mathcal{F}_{11}+A^{3} \mathcal{F}_{31}\right) \cos \omega t+O\left(A^{5}\right) & =\left(F_{0}-F_{90}^{H}-F_{180}+F_{270}^{H}\right) / 4, \\
\left(A^{2} \mathcal{F}_{22}+A^{4} \mathcal{F}_{42}\right) \cos 2 \omega t+O\left(A^{6}\right) & =\left(F_{0}-F_{90}+F_{180}-F_{270}\right) / 4, \\
A^{3} \mathcal{F}_{33} \cos 3 \omega t+O\left(A^{5}\right) & =\left(F_{0}+F_{90}^{H}-F_{180}-F_{270}^{H}\right) / 4, \\
A^{2} \mathcal{F}_{20}+A^{4} \mathcal{F}_{40}+A^{4} \mathcal{F}_{44} \cos 4 \omega t+O\left(A^{6}\right) & =\left(F_{0}+F_{90}+F_{180}+F_{270}\right) / 4 .
\end{aligned}
$$

where the series is truncated to fourth order and the superscript $H$ denotes the Hilbert transform 
185 linear component is directly computed from the linear time history and its Hilbert transform as $A(t)=\sqrt{\eta_{1}^{2}(t)+\eta_{1 H}^{2}(t)}$. The approximated envelopes of the $n^{t h}(n>1)$ harmonics are calculated by raising the linear envelope to the power of $n$ and scaling to the magnitudes of the corresponding 
215

harmonics. The scaling is based on the peak value of the component. It can be seen in Figure 3 that the $n^{\text {th }}$ powered envelopes agree very well with the extracted harmonics, both the shape and the peak phase - consistent with standard wave theory. For comparison, we compute the envelopes directly from the time series of the extracted harmonics as shown in blue dash line. Generally these envelopes show profiles in agreement with those computed from the 'Stokes-like' model. It is however clear that the higher orders are slightly contaminated with noise which is likely to be from sensor error as well as small misalignments in the phasing leading to energy leakage. The noise level remains low and only becomes visible away from the main wave group for fourth and fifth harmonics of the wave elevation. This is likely to be primarily due to sensor noise. The force measurements are made with different sensors so this is not directly relevant to the main results in this paper.

Figure 5 presents the size of the second- and third- order harmonics of the free surface. At the second order theoretical predictions have been made against the analytical results of Dalzell (1999) (who corrected minor issues with Sharma and Dean 1981). There is some scatter which we presume is due to measurement error. There is rather more scatter in the magnitude of the third-order harmonics suggesting either energy leakage between components or measurement noise.

Overall, we conclude that the waves are produced sufficiently accurately for the harmonic decomposition technique to be applied to force. It should be noted that forces (and moments) are more non-linear than the free surface (i.e. higher harmonics play a larger role) and hence are less liable to contamination.

We now turn to consider the total force on the cylinder in the wave direction. Figure 6 shows the decomposed spectra of the horizontal wave force for Case 8 with $k_{p} A=0.21$. The linear force corresponding to the linear free wave component is between $0.5 f_{p}-3 f_{p}$ in the black line with its peak at $f / f_{p}=1$, while the second harmonic is between $1.0 f_{p}-5 f_{p}$ in the red line. The second difference component between $0.0 f_{p}-2.0 f_{p}$ in the blue line can be easily separated from the fourth harmonic as there is little overlap between them. Again, a minor leakage to the first harmonic from the second and third is observed. It is seen that the separation is clean up to $12 f_{p}$. With such cleanly separated spectra, we can extract harmonics up to 12th utilizing appropriate bandpass frequency filters (see Zhao et al. (2017)).

Figure 7 summaries the results in the time domain for a typical case. Firstly, it can be seen that the linear (first order) force's envelope is very similar in shape predicted by using a linear 

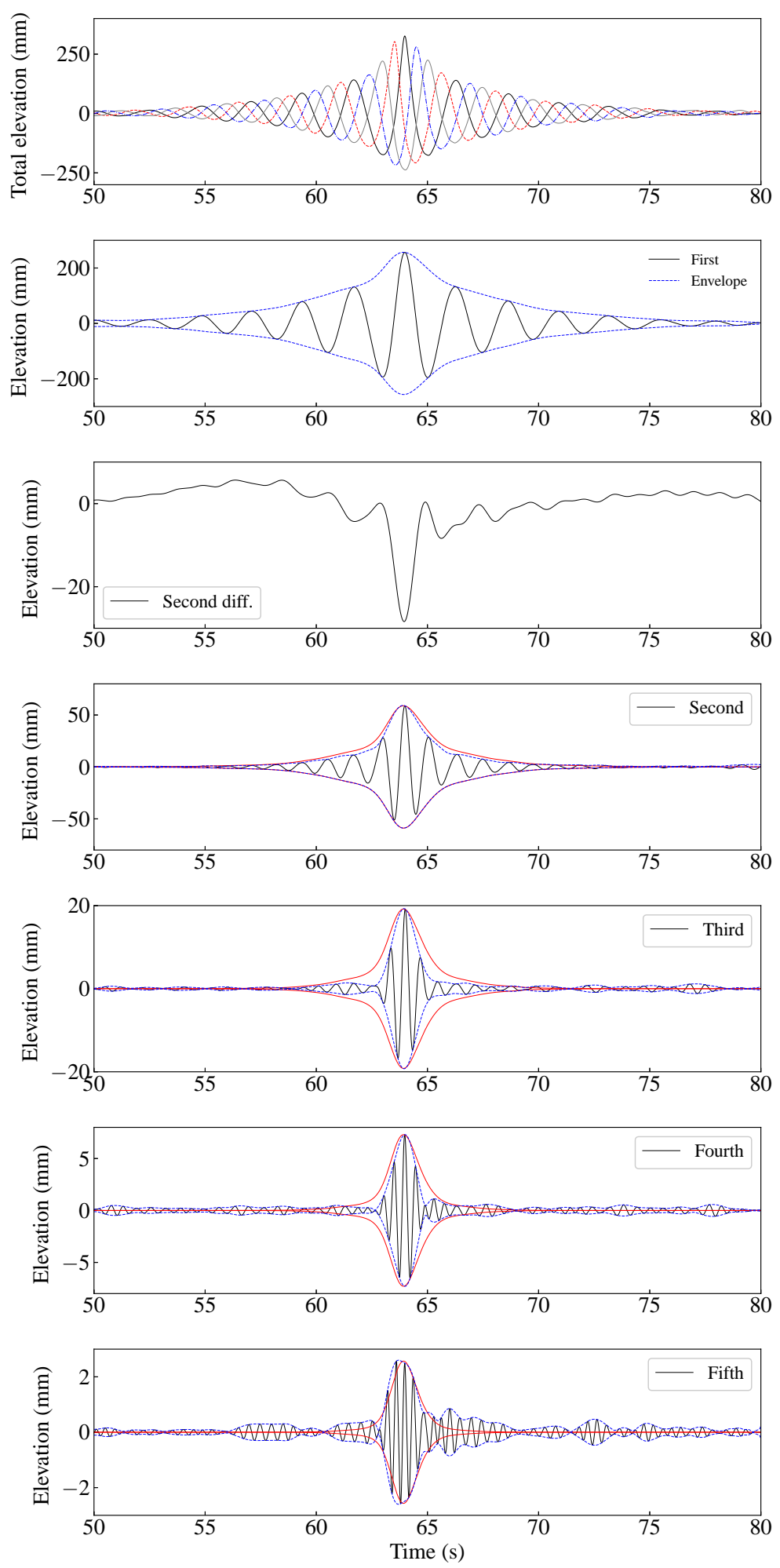

Figure 3: Harmonic components of wave elevation at the centre of the tank for Case $8, f_{p}=0.429 \mathrm{~Hz}$ and $k_{p} A=0.21$. The top panel is the total wave elevation for four phases. The envelopes of the components are in solid red. The linear envelope is directly computed from its time history. Envelopes of higher harmonics are obtained by raising the linear envelope to $n^{\text {th }}$ power and scaling to its magnitude. 


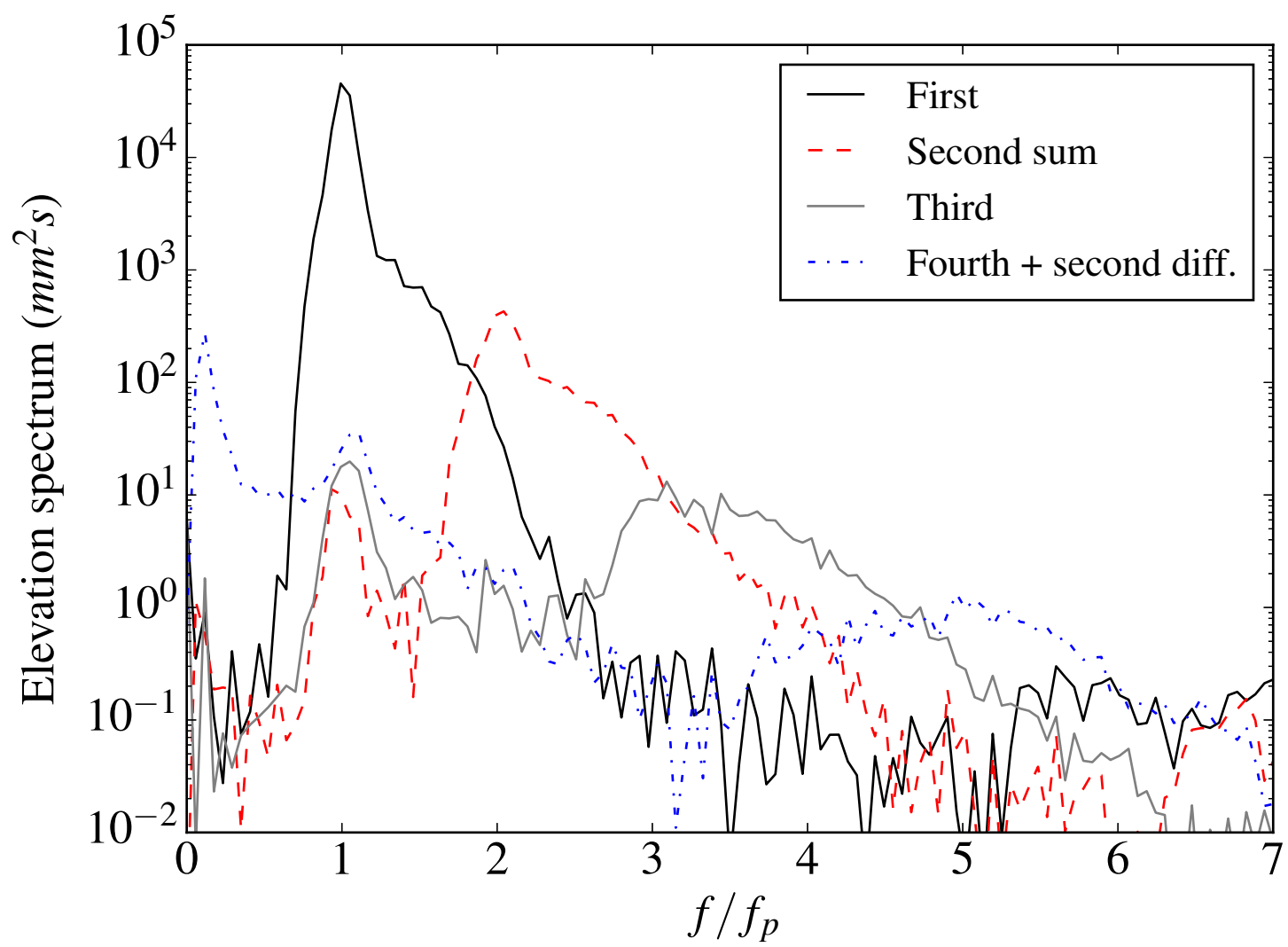

Figure 4: Energy density spectra of wave elevation from four-phase combination for Case $8, f_{p}=0.429 \mathrm{~Hz}$ and $k_{p} A=0.21$. The frequency is normalized by $f_{p}$ and the spectral density is shown on a log scale'.

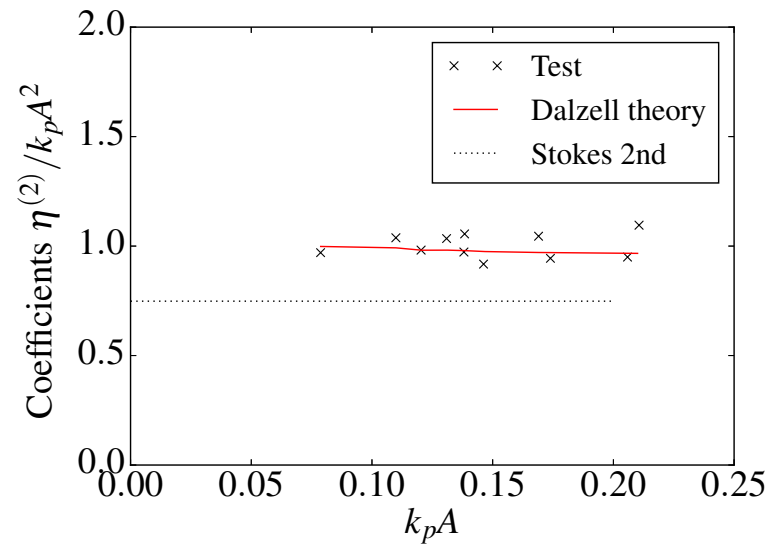

(a)

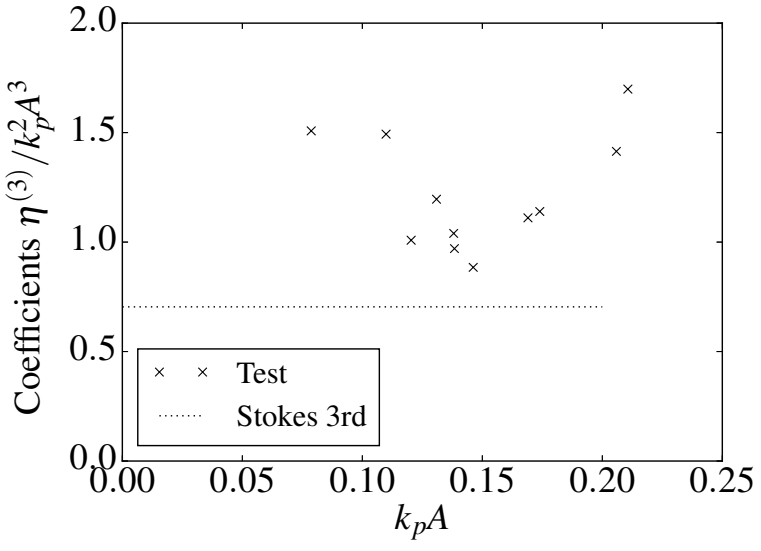

(b)

Figure 5: The non-dimensionalized coefficients for harmonic wave elevations with $f_{p}=0.429 \mathrm{~Hz}$. (a) Second order; (b) Third order. The spectral peak frequency $f p$ is used here for Stokes regular wave theory. 
wave input to Morison's equation. The second order difference force is small and appears to be a combination of the force due to the second order free long wave and a larger skew-symmetric loading due to the return current under the wave-group. Again, we include the envelopes of higher harmonics obtained by the Stokes-like model, i.e. raising the linear force envelope to $n^{\text {th }}$ power and scaling the peak to the measured peak value. The second harmonic agrees almost perfectly with the envelope. The third harmonic force shows some inconsistency. It has a steep rise before focusing and a slow decay. Notably the third harmonic peak force is smaller than the fourth consistent with the work of Ma et al. (2009). The third harmonic, consistent with the previous simulations of Fitzgerald et al. (2014), is however not always well captured by the Stokes-like model. For this harmonic, the agreement with the model varies significantly depending on the case. We have no physical explanation for why the third order appears to agree less well with our model than higher orders. This is not a viscous effect since it appears in potential flow simulations. We have some evidence that there may be two processes going on at this frequency which sometime cancel and sometimes superimpose. But we stress this is speculation and we have not been able to fully interpret our results using this idea. Kristiansen and Faltinsen (2017) found that simply adding viscous effect (which is not considered in the numerical simulation and the theoretical model) did not mitigate the discrepancy between measurement and prediction at the third harmonic. In an experimental work, Molin et al. (2005) showed the significance of the third order effect of the diffracted wave from the cylinder interacting with the incoming wave. Although there is no obvious evidence from the present tests on this tertiary interaction, the high-frequency (third order) diffracted component is clearly captured and might play a role in the behaviour of the third harmonic force. Higher harmonics appear to be extracted cleanly with some structure still discernible at the 12th harmonic. This clean extraction is consistent across all the tests run in the experimental campaign.

The clean extraction of the harmonics allows the empirical model that the forces follow a Stokes like expansion to be examined. If this model is correct the envelope of the harmonics should follow the envelope of the linear force component raised to the appropriate power. For components excluding the third the agreement is typically good across all tests until the forces are small enough that they are seriously contaminated by sensor noise.

The harmonic extraction approach described above is equally good for moments. 


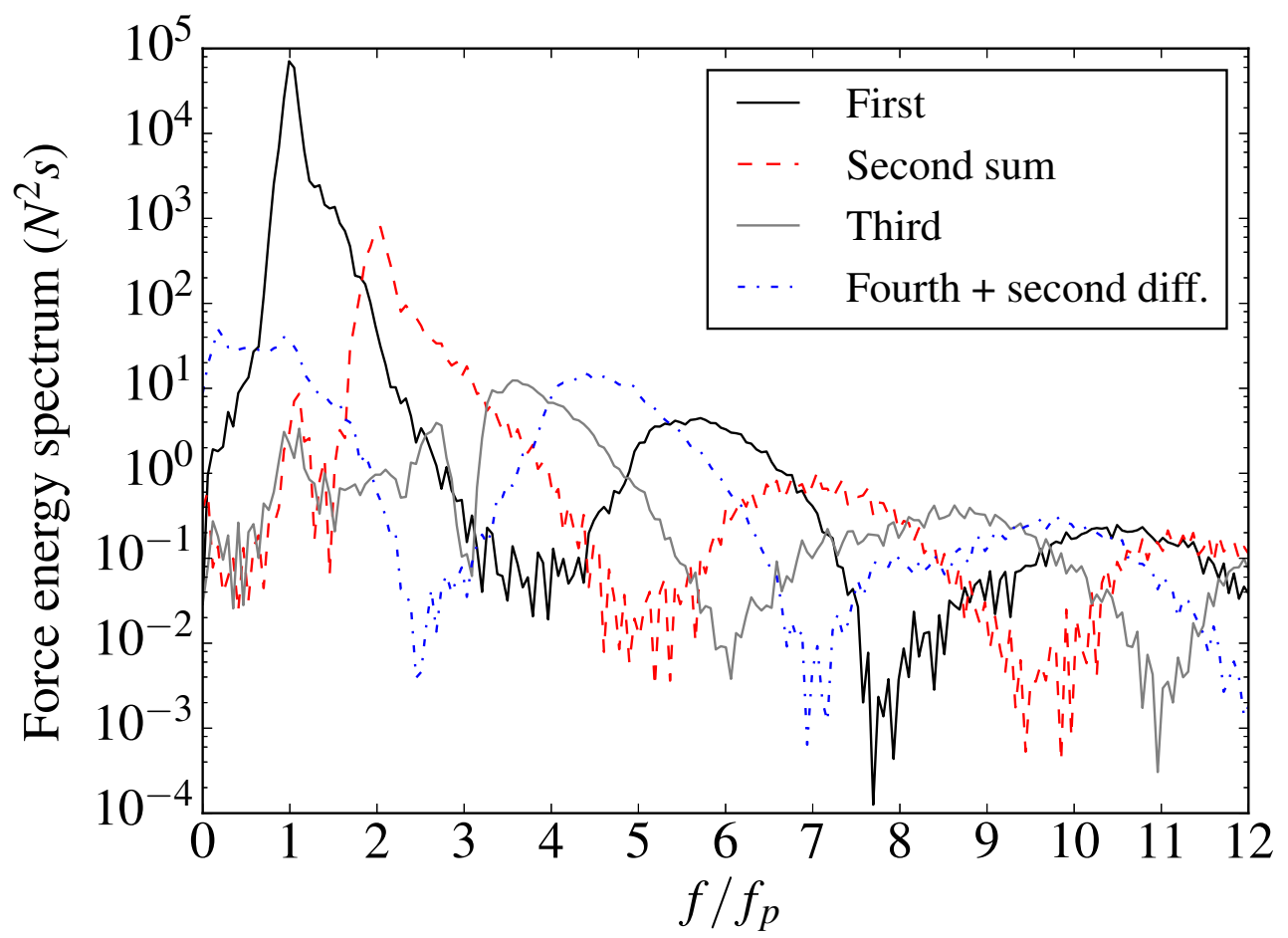

Figure 6: Experimental spectra of horizontal wave force from four-phase combination for Case $8, k_{p} R=0.129$ and $k_{p} A=0.21$. The frequency is normalized by $f_{p}$ and the spectral density is shown on a log scale.

\subsection{Numerical results}

For the numerical experiments controlling the paddle to change the phase of the input is trivial and so we do not repeat the above results showing the free surface. However, unlike for the experiments, it is important to verify the accuracy of the model and determine its limitations. We note that simulating high orders of force necessitates using a very high density free surface and body mesh so as to resolve the relevant small wavenumbers. A detailed description of the verification cases undertaken for the present work is presented in Feng et al. (2019). Selected results from this are presented here.

Mesh convergence is tested by varying the size of a typical element of the mesh based on the wave length of the incident wave, $\lambda$, at the spectral peak frequency $f_{p}$. Three resolutions were considered with element size $\lambda / 30, \lambda / 48$ and $\lambda / 60$ which are described as 'coarse', 'normal' and 'fine'. Note that around the body of the cylinder the element size is much smaller typically being $\lambda / 300$. Figure 8 shows the comparison of the horizontal wave force for the three mesh configurations with $f_{p}=0.429 \mathrm{~Hz}$ and $A=0.06 \mathrm{~m}$. The loads are non-dimensionalized by $\rho g R^{2} A$. We see almost identical time histories of the forces for the three meshes in Figure $8(\mathrm{a})$. 

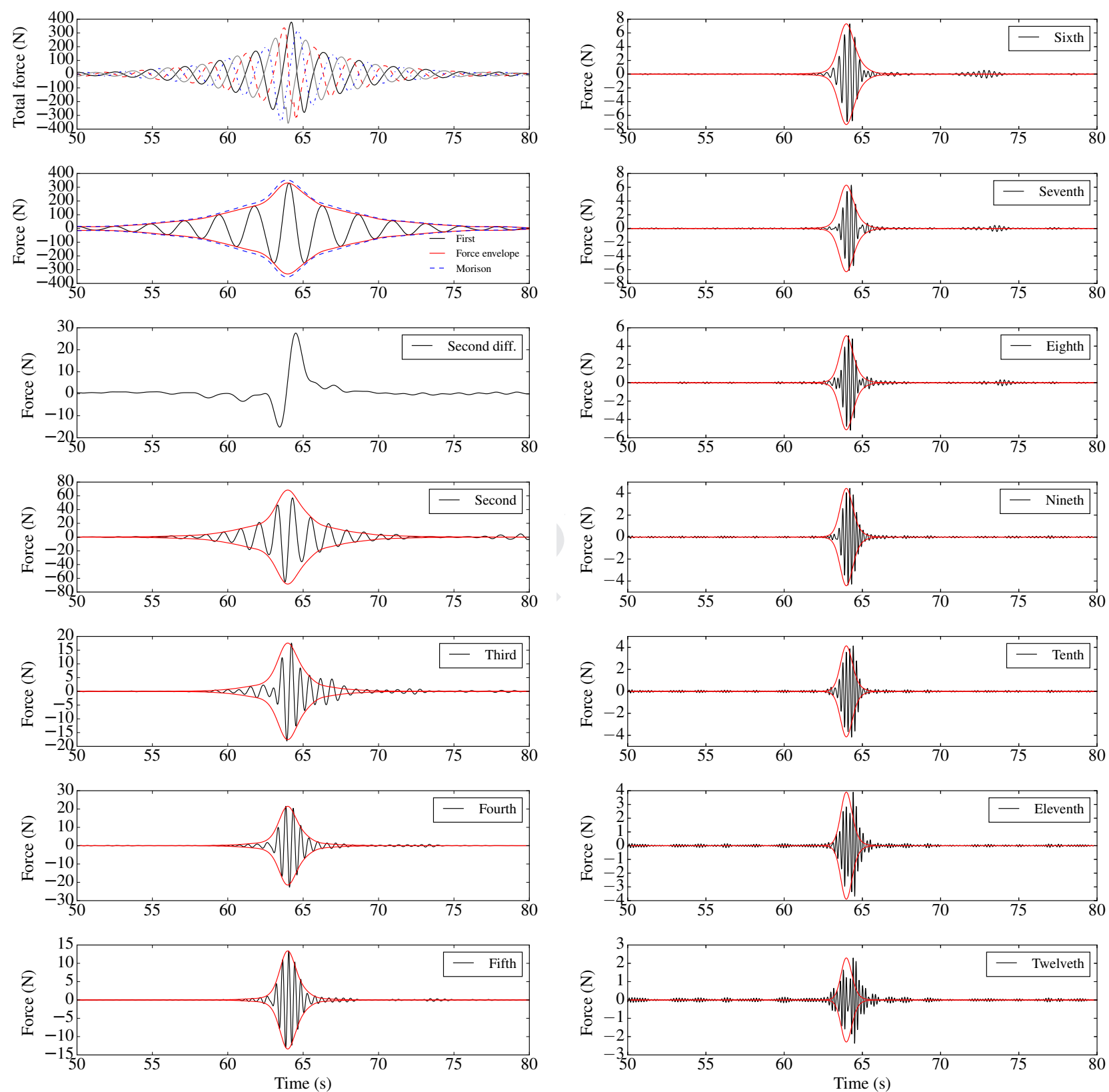

Figure 7: Harmonic components of wave force for Case $8, k_{p} R=0.129$ and $k_{p} A=0.21$. The top left panel is the total wave force for four phases. The envelopes of the components are in solid red. The linear envelope is directly computed from its time history. Morison inertial force computed using linear elevation is in blue dashed line. Envelopes of higher harmonics are obtained by raising the linear force envelope to $n^{\text {th }}$ power and scaling the peak to the measured peak. 


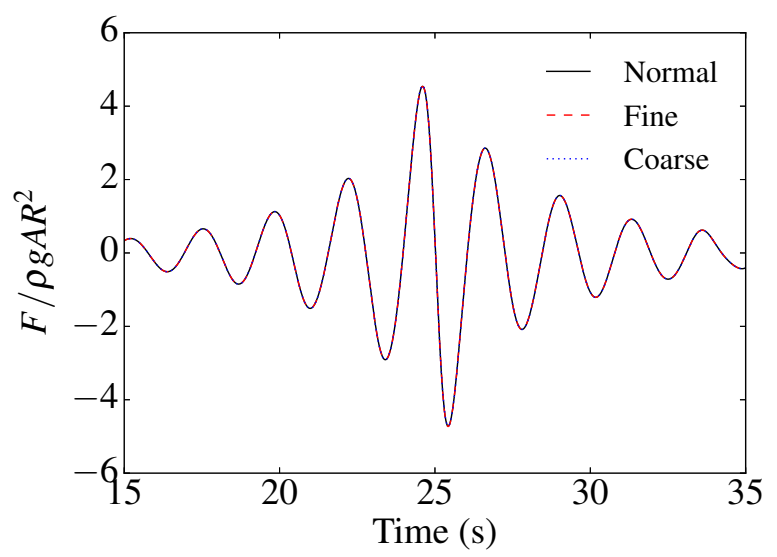

(a)

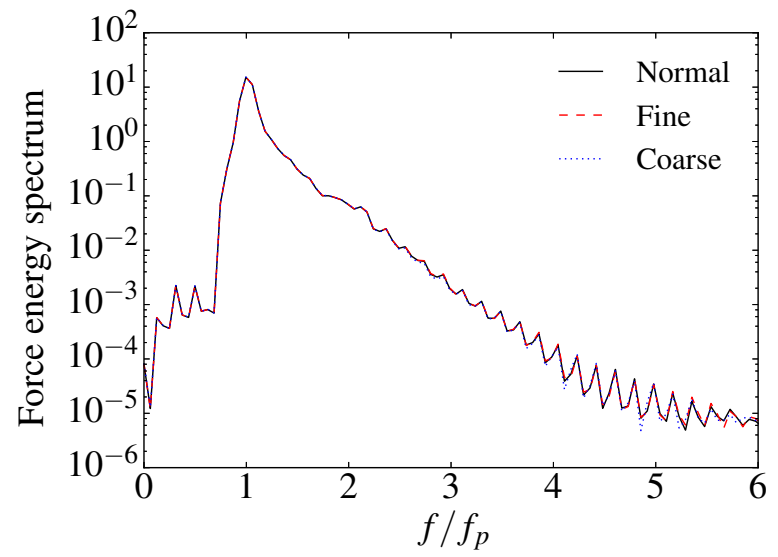

(b)

Figure 8: Numerical convergence of the wave forces on the cylinder for $k_{p} R=0.129$ and $k_{p} A=0.05$. The horizontal wave force is non-dimensionalized by $\rho g R^{2} A$. (a) Time history and (b) energy spectrum.

The force spectra are plotted in Figure 8(b). Up to third order, no difference is visible from the spectra. However, small discrepancies can be seen at the fourth and higher orders between âCoarseâ and the other resolutions. We use a mesh density similar to the âNormalâ configuration in our simulations.

A severe test of the accuracy of the numerical solver (for a non-dissipative model) is to run the simulation forward and then reverse the time step and run the simulation backwards and see if the initial conditions are recovered. In Figure 9 we present the free-surface level at the front stagnation point of the cylinder for forward and backward runs. The difference of the normalized elevation between the forward and backward runs is shown in Figure 3.2b. Given the non-linearity of the problem we consider the agreement between these to be excellent. Other elevation and pressure data show equally good agreement.

As with the experimental results, Figure 10 presents a typical spectrum showing the decomposition into harmonics. This is significantly 'cleaner' than for the experimental data. However, it is clear from this that the beyond fourth harmonic the resolution of the simulations is starting to break down.

Figure 11 presents the harmonic decomposition of numerical results in the time domain. We also present experimental results for the same case although we note that this case was close to the steepness limit at which the numerical model went unstable. The clean decomposition of the harmonics can be seen clearly. The linear force shows excellent agreement between numerics and experiments. The agreement beyond this is less good. The discrepancy in the second order 


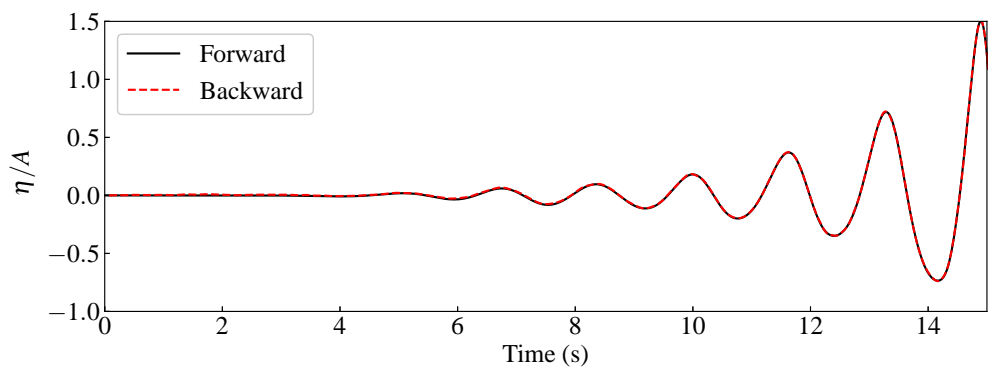

(a)

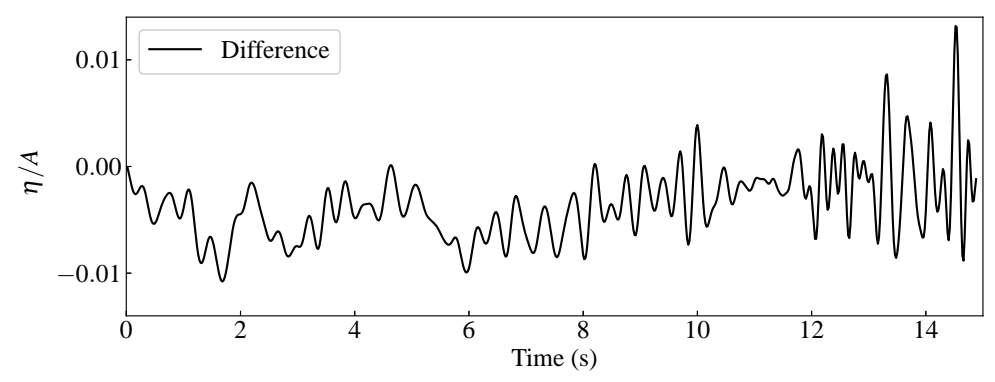

(b)

Figure 9: Elevations at the front stagnation point of the cylinder for time-reversal simulations (a) time history showing forward and backward runs (b) difference between forward and backward runs.

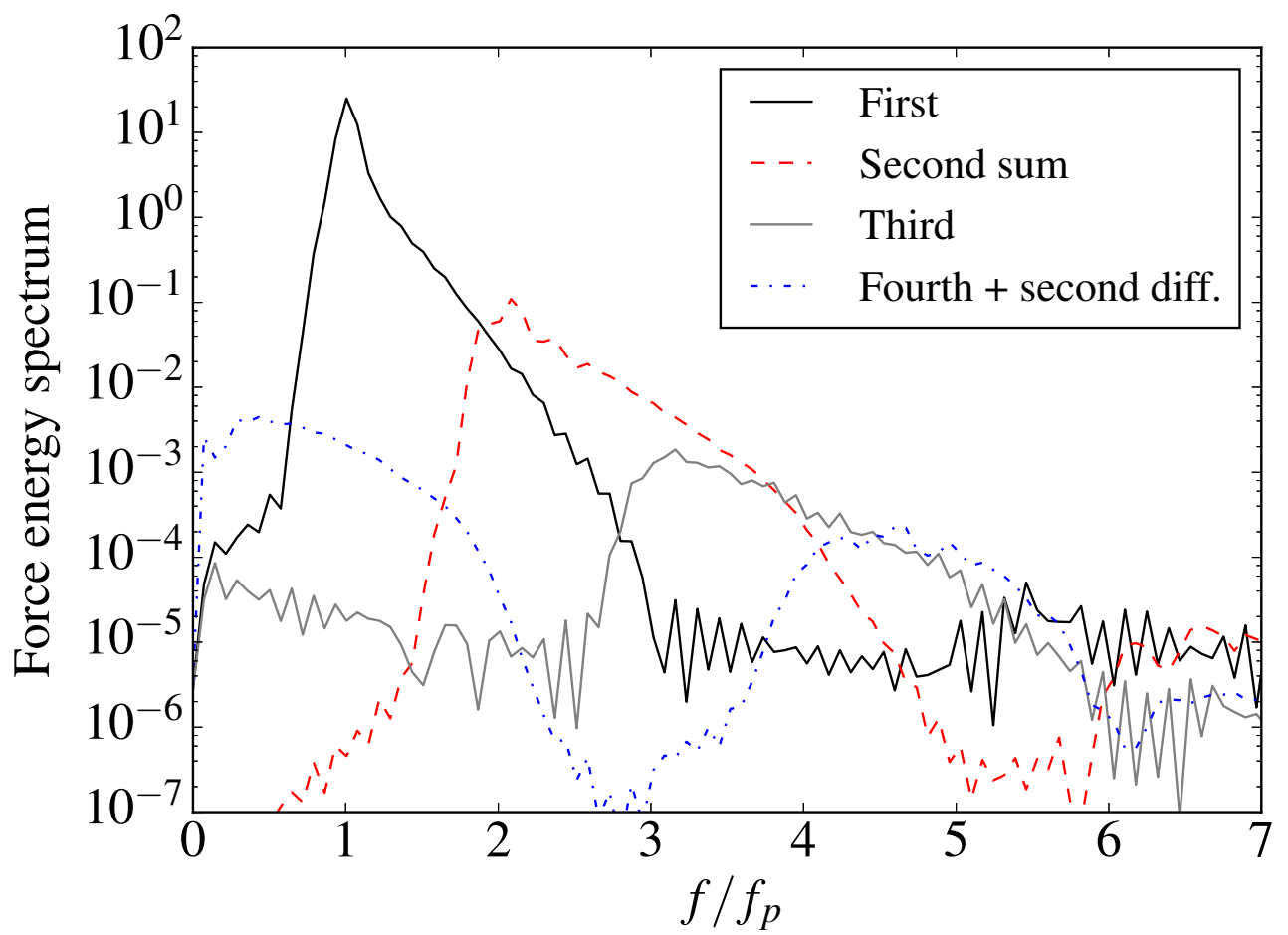

Figure 10: Numerical force spectra of separated harmonics in log-scale with application of the phase-manipulation approach for $k_{p} R=0.129$ and $k_{p} A=0.110$. The horizontal wave force is non-dimensionalized by $\rho g R^{2} A$. 
difference term, which is expected to act almost uniformly over the cylinder, can be explained by the experimental cylinder not going to the bottom of the tank as the numerical cylinder does. The discrepancies at the other harmonics are more of a concern, although there is excellent agreement at the 4th harmonic. The third harmonic, as discussed above, is known to have unusual features: the discrepancy presented here is very significant.

\section{Force}

In this section we consider the harmonics of the inline force acting on the cylinder. Figure 12 presents a summary of the amplitude coefficient of the different harmonics with Figure 13 presented the phase of the component relative to the linear force. 'Grue' scaling is used, thus the amplitude of the $n^{\text {th }}$ harmonic is non-dimensionalised by $F^{(n)} / \rho g A^{n} R^{(3-n)}$ (Huseby and Grue, 2000). The harmonics extracted from both experiments and numerics were based on the peak of the envelope of the harmonic.

Theoretical calculations are included at the second order (Chau and Eatock Taylor, 1992;

Faltinsen et al., 1995) and at third order for FNV. The linear code is the result calculated from a potential flow model based on the linear panel method. We include on our plot the result from the experimental study of Huseby and Grue (2000) (H\&G). Their tests were for regular waves with $k R=0.166$. The water depth of these tests was $0.6 \mathrm{~m}$ which, due to their methodology means their water depth varied between experiments (from a max of $k h=3.32$ to $k h=5.67$ ). This compares to the value in our test of $k_{p} h=1.476$. Our tests also used wavegroups which have a non-zero although relatively narrow bandwidth. We do not expect this to make a significant difference to the harmonic coefficients (other than for the 'difference' term). We note that the H\&G tests typically agree rather well with those of Stansberg (1997) and so for clarity we simply present these.

The linear force coefficients show good agreement with theory with little variation as a function of wave steepness. The second, fourth and fifth harmonic coefficients show relatively small variations with $k_{p} A$ suggesting the Stokes-like model is reasonable. At the second order there is reasonable agreement with the analytical results of Chau and Eatock Taylor (1992) for both amplitude and phase. The experimental data in particular shows excellent agreement. As described in the literature (Fitzgerald et al., 2014; Chen et al., 2018), and above, the third harmonic consistently shows the most variability compared to the other harmonics. This is true both between different data sets and between the different numerical and experimental results. Fourth and fifth 

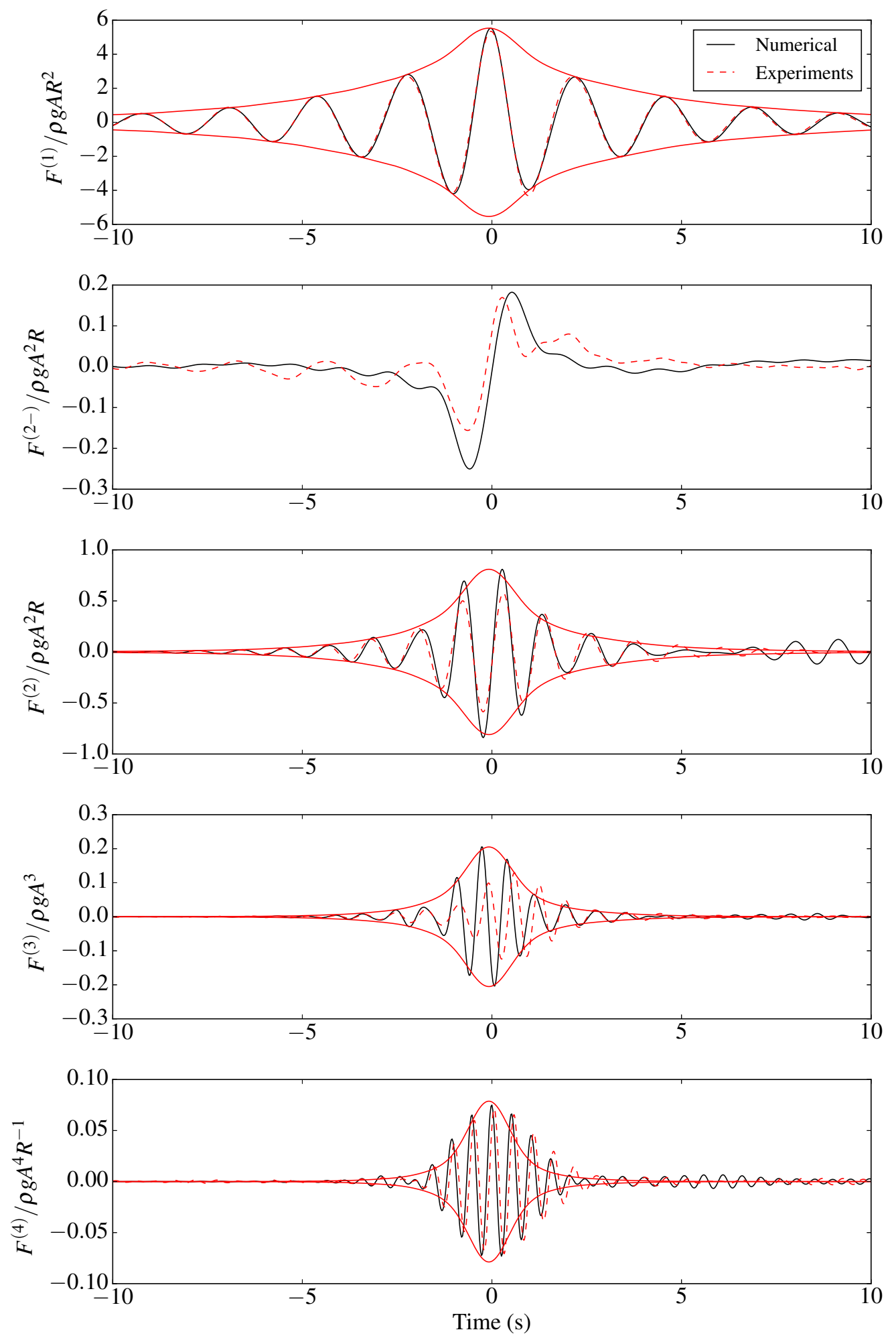

Figure 11: Comparison of decomposed harmonic wave forces with tests using the phase-manipulation approach for Case $1, k_{p} R=0.129$ and $k_{p} A=0.110$. The forces are non-dimensionalized by $\rho g A^{n} R^{(3-n)}$. The envelopes of $n$th harmonics are obtained by raising the linear force envelope to its $n$th power with proper scaling. 


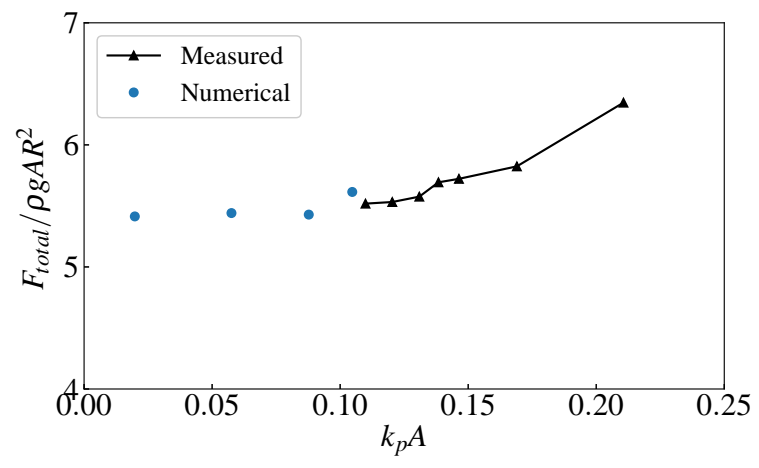

(a)

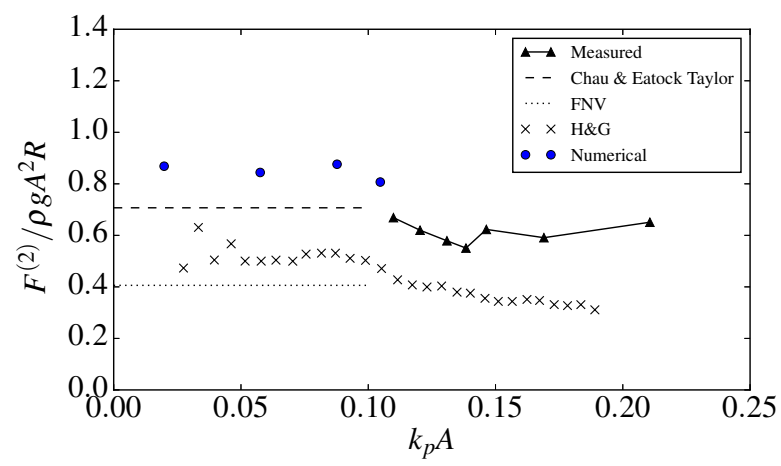

(c)

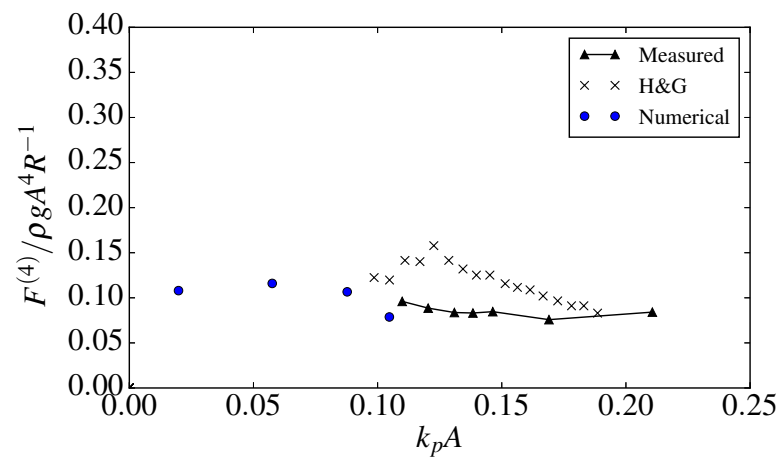

(e)

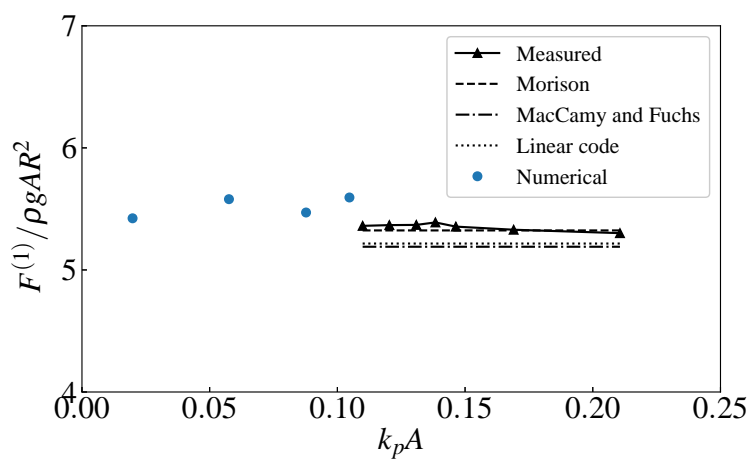

(b)

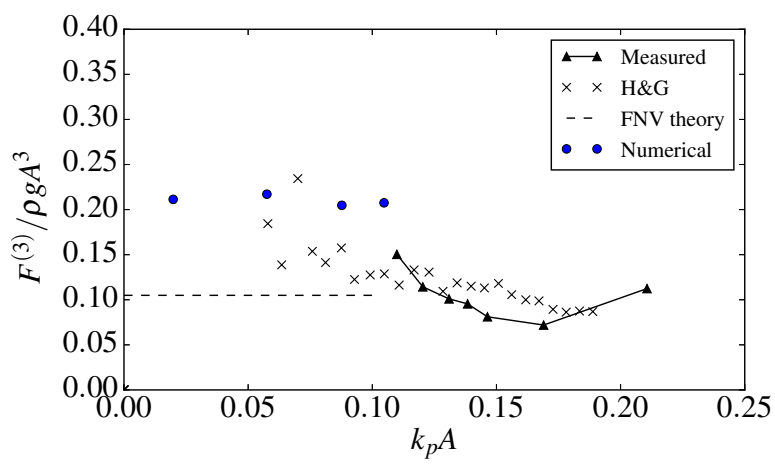

(d)

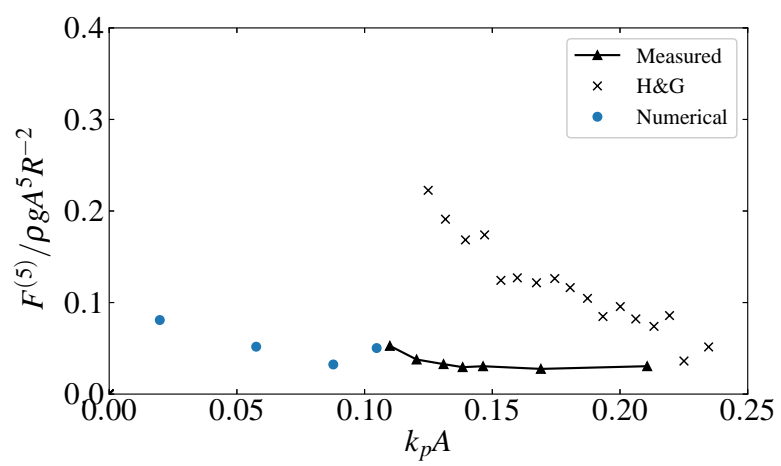

(f)

Figure 12: Variation of harmonic forces with increasing wave steepness $k_{p} A$. The $n^{\text {th }}$ harmonic force $F^{(n)}$ is non-dimensionalized by $\rho g A^{n} R^{(3-n)}$. The solid lines are the present measured results. The Huseby and Grue (2000) (H\&G) results are for regular wave tests with $k R=0.166$ in a water depth $0.6 \mathrm{~m}$ and $R=0.03 \mathrm{~m}$. Chau and Eatock Taylor (1992) results are from the second-order diffraction theory. 


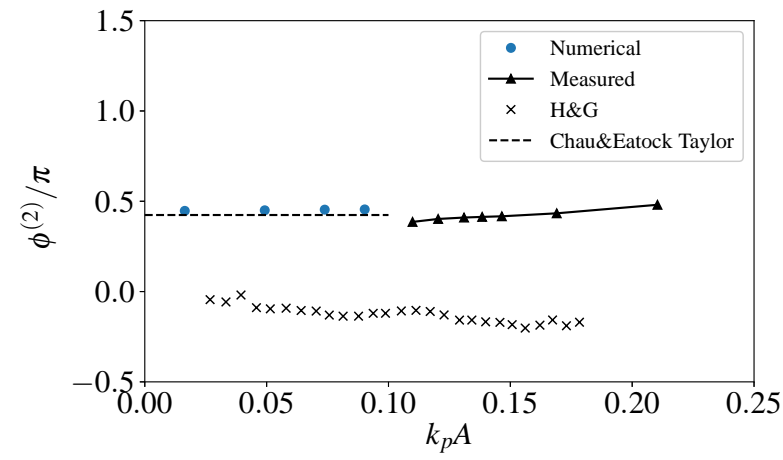

(a)

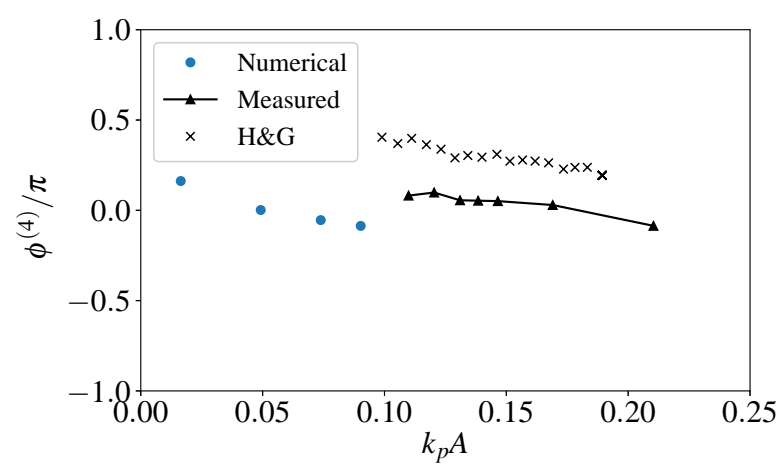

(c)

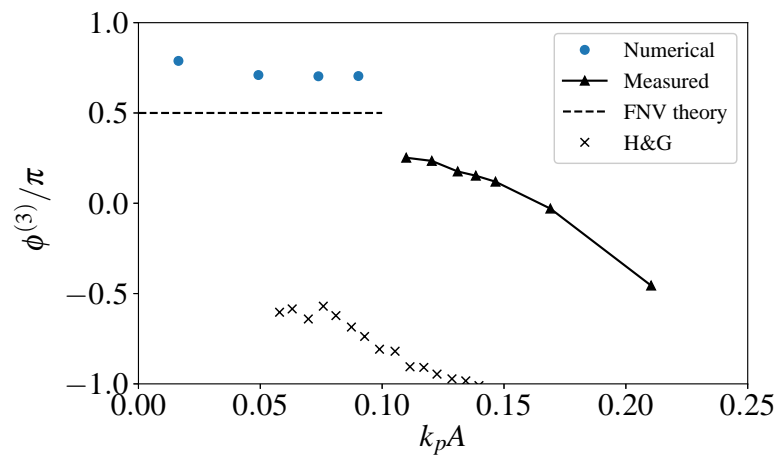

(b)

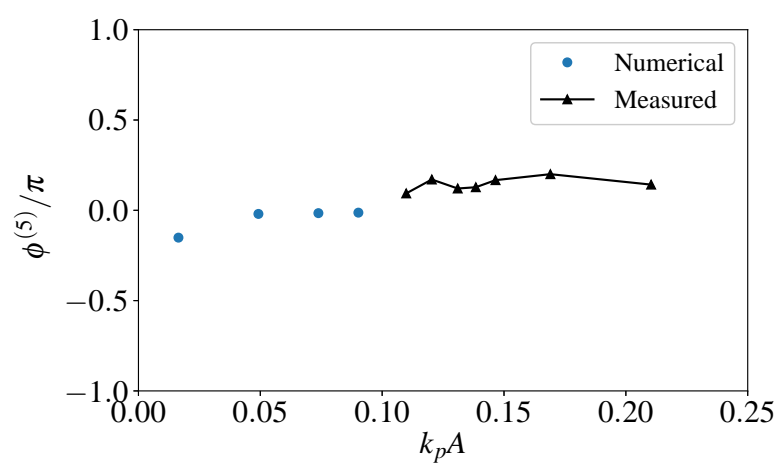

(d)

Figure 13: Variation of harmonic force phases with increasing wave steepness $k_{p} A$ for $f_{p}=0.429 \mathrm{~Hz}\left(k_{p} R=0.129\right)$.

harmonics show good consistency across the range of steepnesses suggesting that the approach works well for these.

The harmonic decomposition works reasonably well for cylinders in the 'inertia' range typical for offshore wind turbines. Figure 14 presents numerical results using the same methodology for a larger cylinder with $k_{p} R=1.0$ (so approximately eight times that in the above analysis) and $K C=0.4$. The discrepancy between the scaled envelope and the extracted harmonics shows that once diffraction effects become significant the assumed Stokes-like model might not be appropriate, though the Stokes expansion remains applicable for low steepness waves. This is consistent with the expectations and the findings of Chen et al. (2018) and Chen et al. (2019). The measured individual nonlinear force contributions occur earlier in time than is assumed in our Stokes-like model. This would be consistent with these harmonics being driven principally by wave-structure interactions on the upwave half of the cylinder.

We now examine the third harmonic force component and its relationship with the radius of the cylinder. Figure 15 presents the magnitude of the third order harmonic as a function of the 

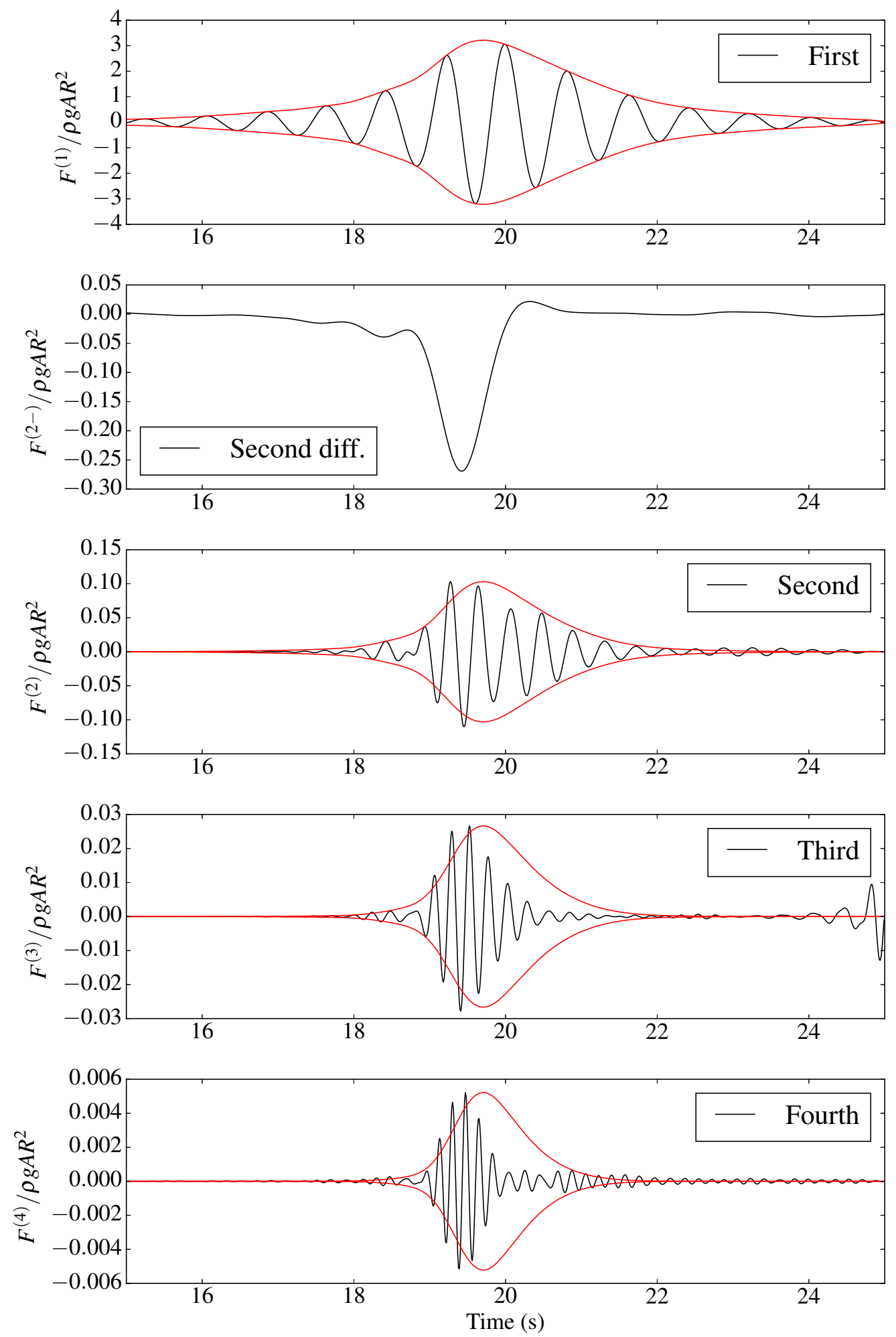

Figure 14: Decomposed numerical harmonic wave forces for $f_{p}=1.26 \mathrm{~Hz}\left(k_{p} R=1.0\right)$ and $A=0.02 \mathrm{~m}$. The forces are non-dimensionalized by $\rho g A^{n} R^{(3-n)}$. The envelopes of $n$th harmonics are obtained by raising the linear envelope to $n$th power with proper scaling. 


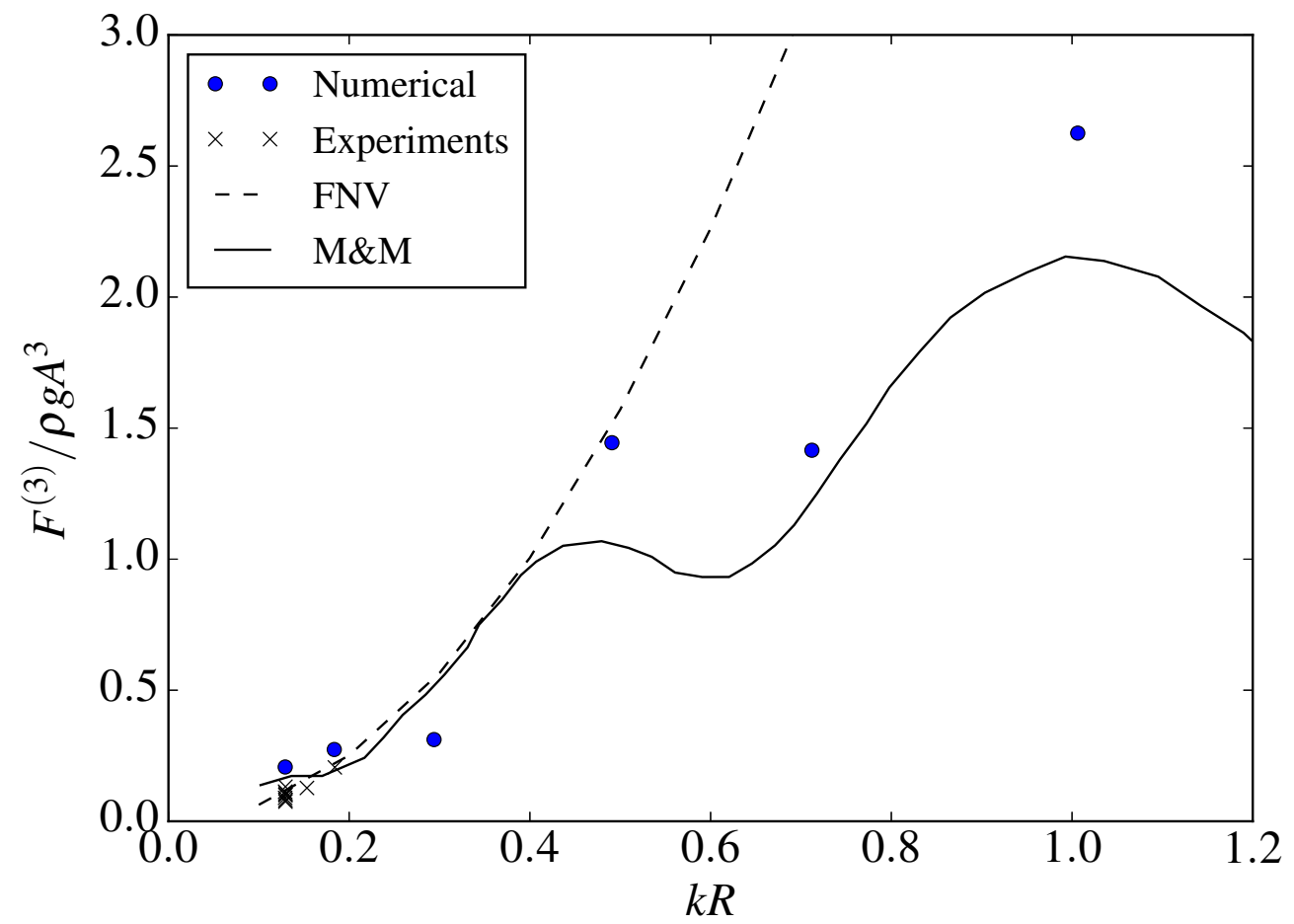

Figure 15: Third harmonic forces over $k_{p} R$. For the experimental and numerical results the envelope peak values are utilized.

non-dimensionalised radius. We only have experimental results for relatively slender cylinders. Our data is compared to the analytical results of FNV theory and M\&M. The former assume the cylinder to be slender and so agreement would only be expected for relatively small $k R$. The theoretical predictions of M\&M however show reasonable qualitative agreement across the range of values studied here although there are significant quantitative differences. Likewise, the fifth harmonic force also acts close to the mean water surface, now slightly higher than the fourth, so apart from a data point (case 4) now slightly above still water.

\section{Moment}

We now turn to examining moments in detail. We choose to analyse the moment acting around the base of the column (i.e. the 'mudline') as this is of most significance to practical engineering design. Unlike forces, there are only a few studies which directly examine moments, presumably due to the difficulty in accurately measuring these experimentally.

To evaluate the nonlinear effects on the higher harmonic moment, we non-dimensionalise for the $n^{t h}$ harmonic of moment using $\rho g A^{n} R^{3-n} h$ which adds the mean water depth to the scaling of force introduced by Huseby and Grue (2000). The variation of the non-dimensionalized peak 


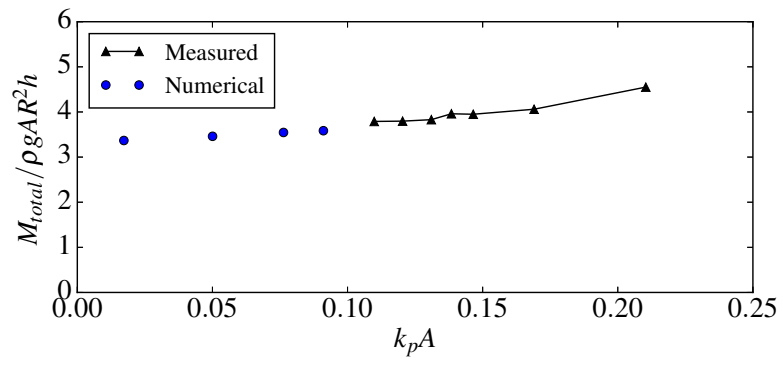

(a)

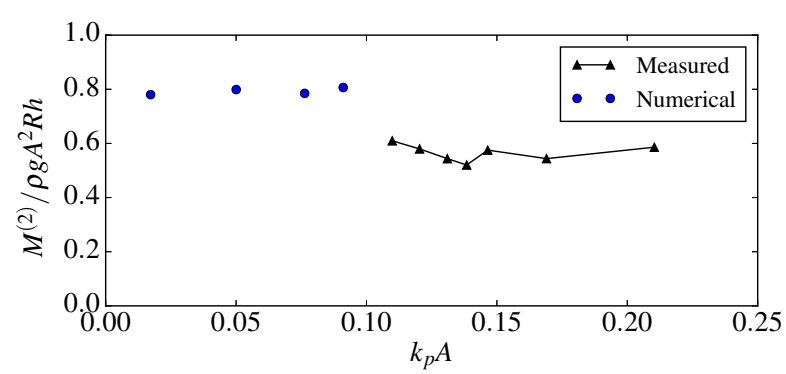

(c)

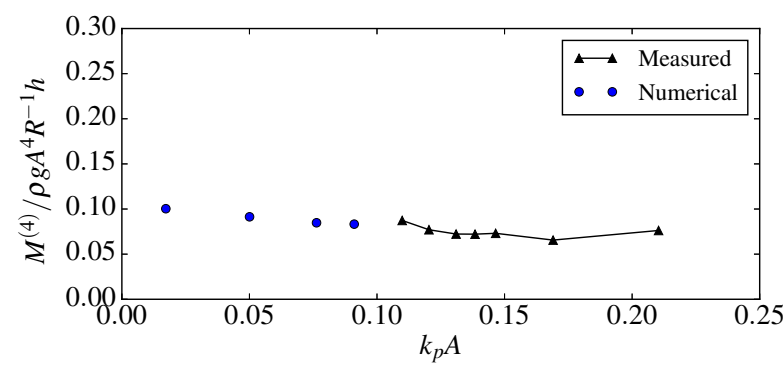

(e)

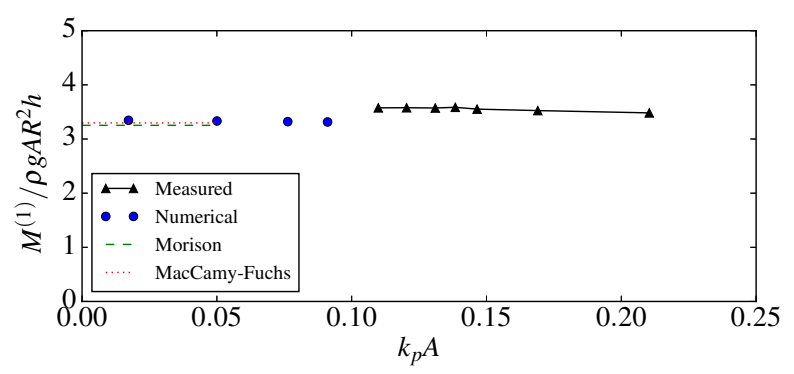

(b)

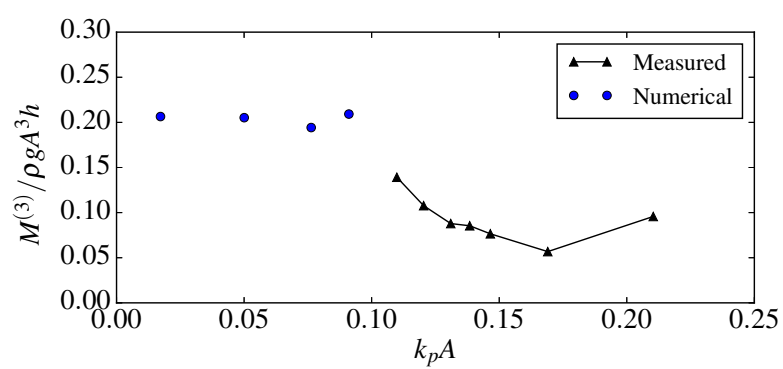

(d)

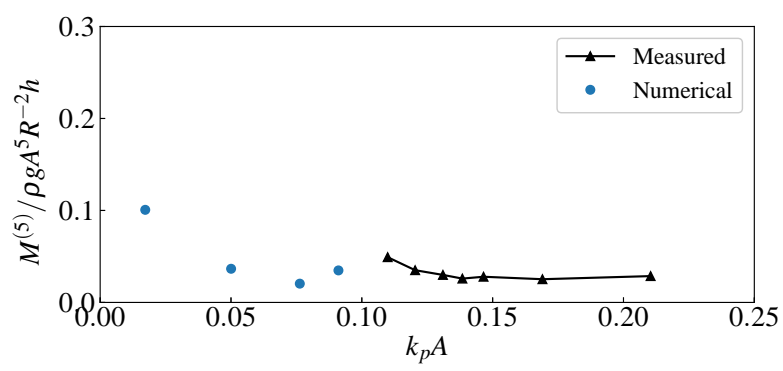

(f)

Figure 16: Variation of peak harmonic mudline moments with increasing wave steepness $k_{p} A$. The $n^{\text {th }}$ harmonic moment $M^{(n)}$ is non-dimensionalized by $\rho g A^{n} R^{(3-n)} h$. The solid lines are the present measured results.

harmonic moment against increasing wave steepness is plotted in Figure 16. The overall trend for each harmonic component is similar to that of the horizontal force. Except for the third harmonic, the non-dimensionalized moment coefficient is almost a constant value with increasing steepness $k_{p} A$. However we notice that the numerical results slightly over-predict the measurements for the second and third harmonics. The reason might be that slight energy 'leakage' occurs from the second and third harmonics to the first in the tests, as can be seen from the spectra in Figure 6, while no leakage is present in the numerical simulation in Figure 10.

To understand the moment values further, it is useful to consider an effective point on the cylinder at which the force 'acts'. The force is, of course, oscillatory and therefore we consider 


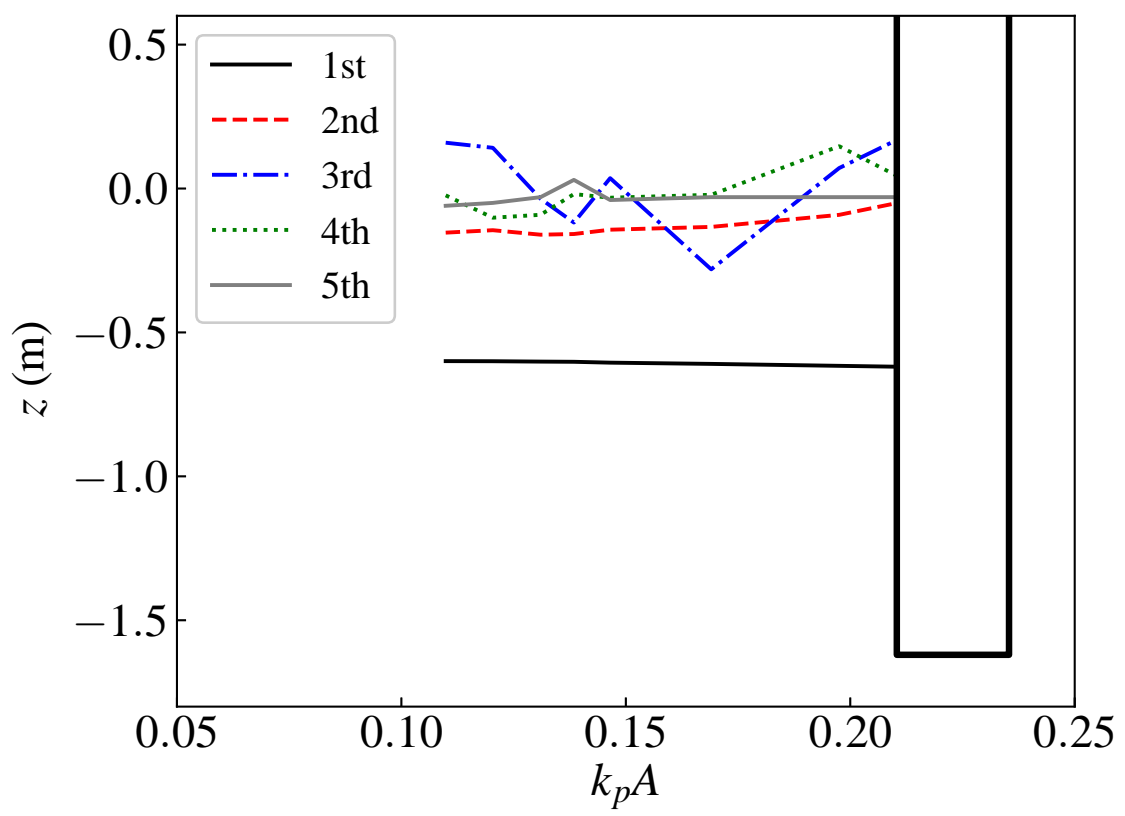

Figure 17: The moment arm of each harmonic force on the cylinder for $k_{p} R=0.129$. The vertical axis shows the z-coordinate along the cylinder. The bold solid line represents the cylinder.

the ratio of the envelope of the moment to the envelope of the force,

$$
L_{n}=\frac{\max \left(\sqrt{M_{n}^{2}+M_{n H}^{2}}\right)}{\max \left(\sqrt{F_{n}^{2}+F_{n H}^{2}}\right)},
$$

and we use the maximum values of envelopes of the harmonic moment and force.

The locations of the moment arm for each harmonic of moment and force in the physical experiments are illustrated in Figure 17. We show the vertical coordinates of the harmonic force locations and the bold solid line represents the cylinder (not in scale). It is seen that the linear force position is constant at $0.6 \mathrm{~m}$ below still water surface with increasing $k_{p} A$, which is about one third of the submerged cylinder and consistent with linear theory. The second harmonic force position is stable as well, with only very small movement closer to the still water surface. The third harmonic shows the most significant variation with the line of action sometimes being above and sometimes below the free surface. This can be compared to FNV theory where at the third order the force can be approximated as a point force at the free surface. The fourth and fifth harmonic forces also act close to the free surface with rather less variation than the third harmonic. 


\section{Nonlinear load estimation based on linear load} linear force time history, a complex coefficient for each force harmonic (so as to model both the magnitude and phase) and an appropriate vertical location for the effective point of action for each force harmonic.

\section{Conclusions} inertia dominated surface-piercing cylinder. Care was taken to ensure that the cylinder was rigid and no significant structural dynamics was excited. High quality experiments are backed up by fully non-linear potential flow numerical simulations. 


\begin{tabular}{|c|c|c|c|c|c|c|}
\hline Case & Harmonics & $\begin{array}{c}\text { Force } \\
\left(/ \rho g A^{n} R^{(3-n)}\right)\end{array}$ & $\begin{array}{l}\text { Phase for force } \\
\qquad(/ \pi)\end{array}$ & $\begin{array}{c}\text { Moment } \\
\left(/ \rho g A^{n} R^{(3-n)} h\right)\end{array}$ & $\begin{array}{l}\text { Phase for moment } \\
\qquad(/ \pi)\end{array}$ & $\begin{array}{l}\text { Moment arm } \\
\quad(/ h)\end{array}$ \\
\hline \multirow{5}{*}{1} & 1 & 5.36 & - & 3.57 & - & 0.67 \\
\hline & 2 & 0.69 & 0.38 & 0.61 & 0.40 & 0.91 \\
\hline & 3 & 0.15 & 0.25 & 0.14 & 0.29 & 1.02 \\
\hline & 4 & 0.096 & 0.081 & 0.087 & 0.08 & 1.01 \\
\hline & 5 & 0.053 & 0.09 & 0.049 & 0.08 & 1.03 \\
\hline \multirow{5}{*}{2} & 1 & 5.38 & - & 3.58 & - & 0.67 \\
\hline & 2 & 0.62 & 0.40 & 0.58 & 0.41 & 0.93 \\
\hline & 3 & 0.11 & 0.23 & 0.11 & 0.29 & 1.04 \\
\hline & 4 & 0.089 & 0.098 & 0.077 & 0.12 & 0.97 \\
\hline & 5 & 0.038 & 0.17 & 0.035 & 0.19 & 1.03 \\
\hline \multirow{5}{*}{3} & 1 & 5.37 & - & 3.57 & - & 0.67 \\
\hline & 2 & 0.59 & 0.40 & 0.54 & 0.41 & 0.94 \\
\hline & 3 & 0.10 & 0.17 & 0.09 & 0.25 & 0.97 \\
\hline & 4 & 0.084 & 0.055 & 0.072 & 0.08 & 0.97 \\
\hline & 5 & 0.033 & 0.12 & 0.030 & 0.16 & 1.02 \\
\hline \multirow{5}{*}{4} & 1 & 5.39 & - & 3.59 & - & 0.67 \\
\hline & 2 & 0.55 & 0.41 & 0.52 & 0.42 & 0.94 \\
\hline & 3 & 0.10 & 0.15 & 0.09 & 0.23 & 0.99 \\
\hline & 4 & 0.083 & 0.053 & 0.072 & 0.08 & 0.97 \\
\hline & 5 & 0.029 & 0.13 & 0.026 & 0.18 & 0.98 \\
\hline \multirow{5}{*}{5} & 1 & 5.35 & - & 3.55 & - & 0.66 \\
\hline & 2 & 0.62 & 0.41 & 0.58 & 0.42 & 0.92 \\
\hline & 3 & 0.08 & 0.12 & 0.08 & 0.22 & 1.04 \\
\hline & 4 & 0.085 & 0.051 & 0.073 & 0.08 & 0.97 \\
\hline & 5 & 0.030 & 0.17 & 0.028 & 0.21 & 1.02 \\
\hline \multirow{5}{*}{6} & 1 & 5.32 & - & 3.53 & - & 0.66 \\
\hline & 2 & 0.59 & 0.43 & 0.54 & 0.44 & 0.92 \\
\hline & 3 & 0.07 & -0.03 & 0.06 & 0.09 & 0.89 \\
\hline & 4 & 0.076 & 0.029 & 0.066 & 0.07 & 0.97 \\
\hline & 5 & 0.028 & 0.20 & 0.025 & 0.26 & 1.02 \\
\hline \multirow{5}{*}{7} & 1 & 5.31 & - & 3.51 & - & 0.66 \\
\hline & 2 & 0.62 & 0.44 & 0.55 & 0.47 & 0.91 \\
\hline & 3 & 0.09 & -0.22 & 0.07 & -0.21 & 0.92 \\
\hline & 4 & 0.082 & -0.041 & 0.070 & 0.06 & 0.98 \\
\hline & 5 & 0.028 & 0.16 & 0.027 & 0.23 & 1.02 \\
\hline \multirow{5}{*}{8} & 1 & 5.30 & - & 3.48 & - & 0.66 \\
\hline & 2 & 0.65 & 0.48 & 0.59 & 0.49 & 0.90 \\
\hline & 3 & 0.11 & -0.45 & 0.10 & -0.51 & 0.95 \\
\hline & 4 & 0.084 & -0.085 & 0.076 & 0.05 & 1.00 \\
\hline & 5 & 0.030 & 0.14 & 0.029 & 0.22 & 1.03 \\
\hline
\end{tabular}

Table 2: Experimentally derived force and moment coefficients from the experiments in this study with $k_{p} R=0.129$, $k_{p} h=1.478, k_{p} A=0.11-0.21$ (see Table 1 for details of the different cases). 


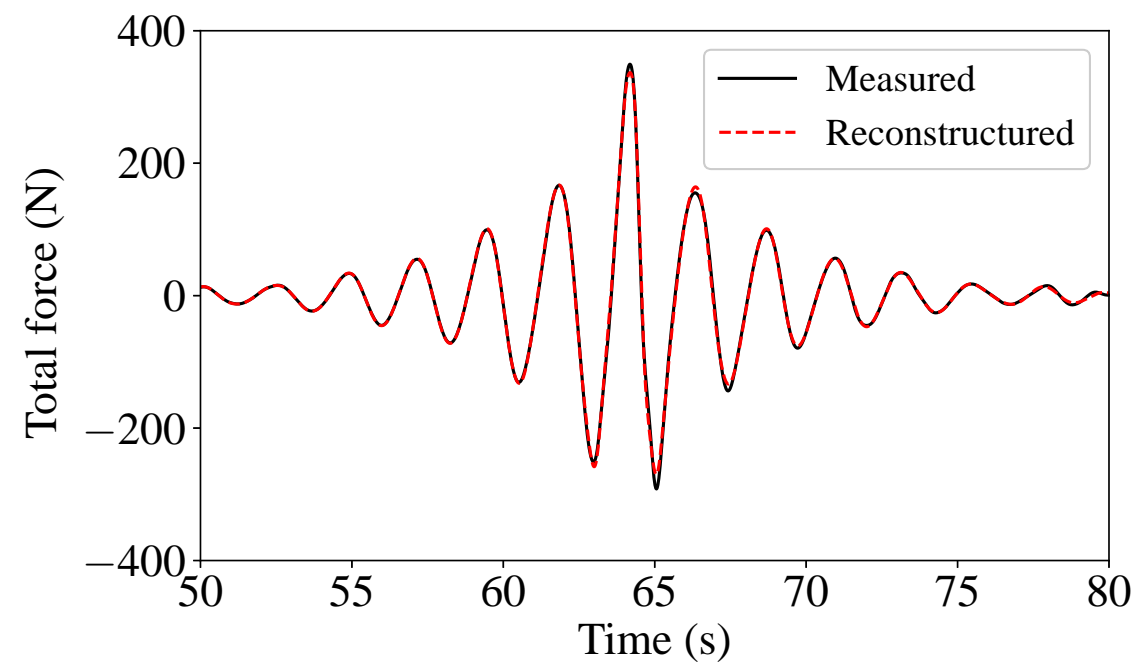

Figure 18: Reconstruction of nonlinear force for Case $8, k_{p} R=0.129$ and $k_{p} A=0.21$.

For cylinders in the 'inertia' range, the Stokes-like model of harmonics for both force and

The simple Stokes-like model for higher harmonics explored in this paper is a good but not perfect physical model for loading on a slender cylinder. However, it does capture much of the structure of the higher harmonics and as such we believe it can serve as a useful, computationally 

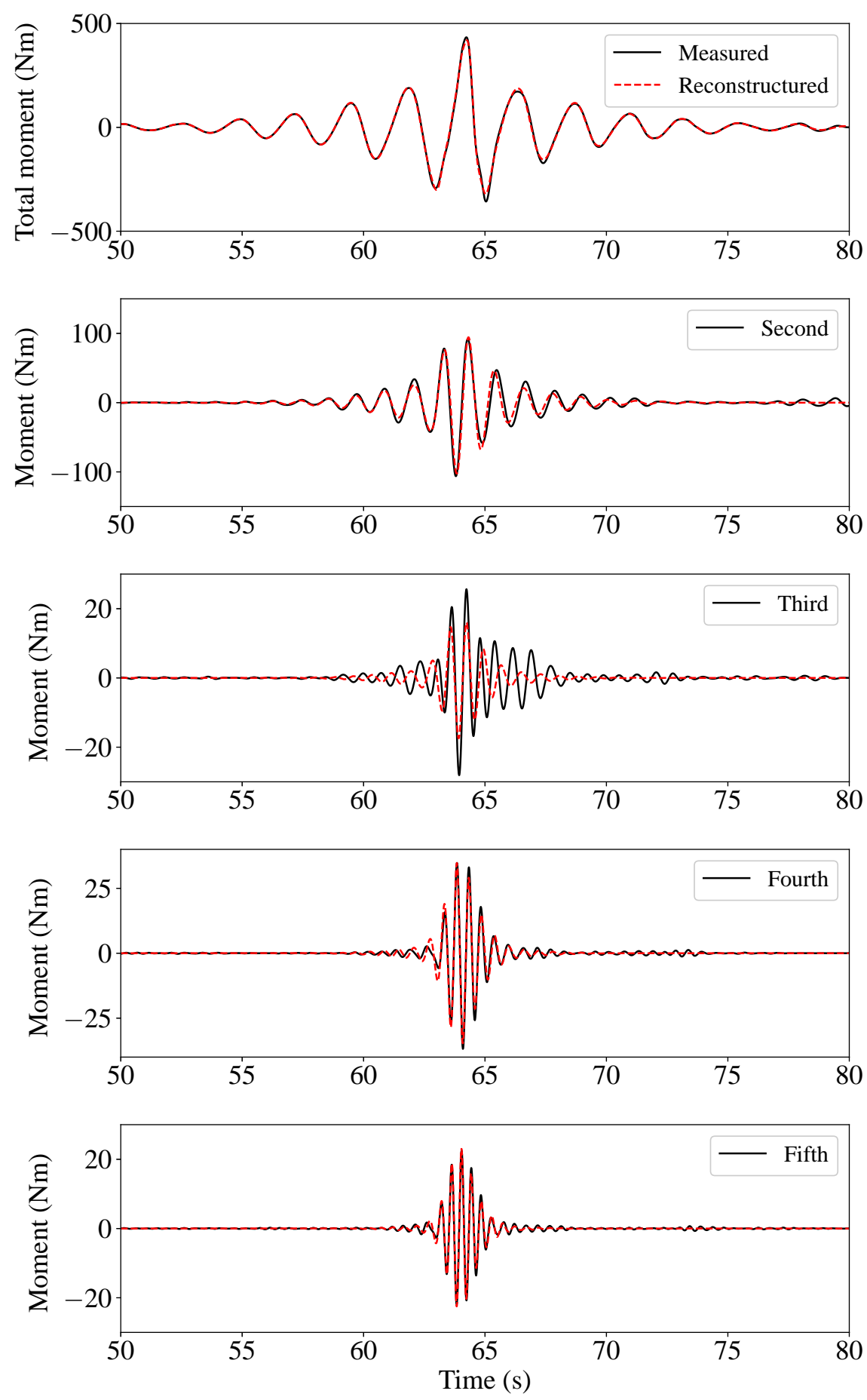

Figure 19: Reconstruction of harmonic moments for Case $8, k_{p} R=0.129$ and $k_{p} A=0.21$. 


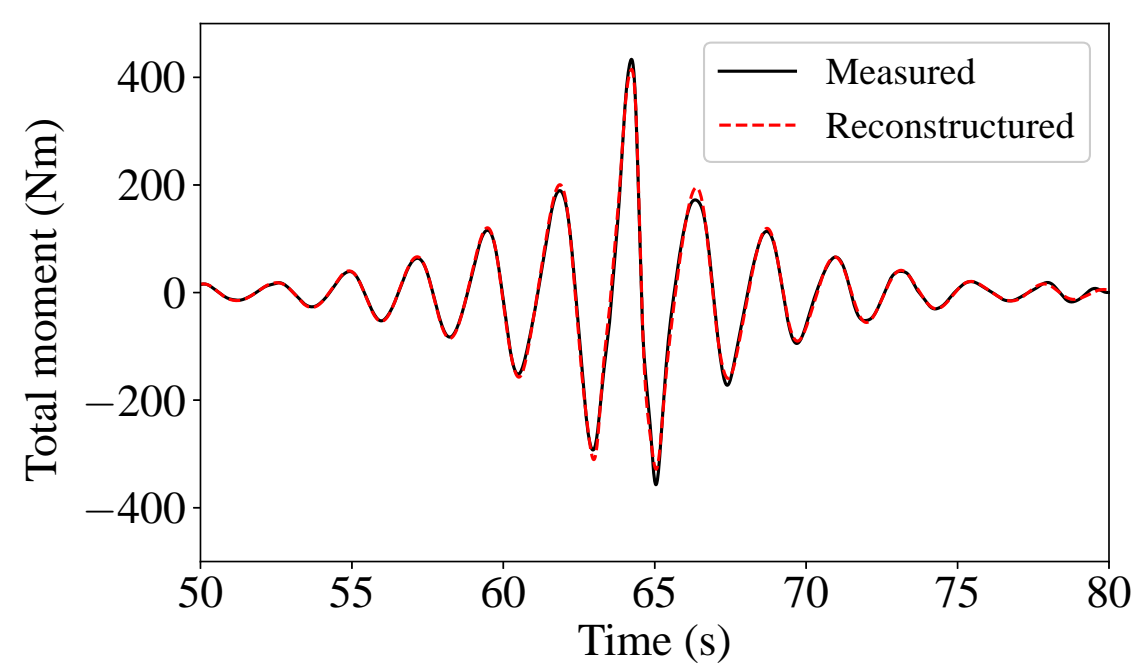

Figure 20: Reconstruction of harmonic moments for Case $8, k_{p} R=0.129$ and $k_{p} A=0.21$. Moments reconstructed from force coefficients multiplied by the effective moment arm.

fast, engineering model. The approach would for instance be able to generate quickly a very long simulations of loads on monopiles such as those currently used in the offshore wind industry. To calculate base shear and moment for a structure a transfer function would have to be employed. Such simulations would be useful for fatigue analysis which in some locations is a design driver for these structures.

\section{Acknowledgement}

This work was funded by UK/China ORE funding (EPSRC grant EP/R007632/1) and the United Kingdom Centre for Marine Energy Research (UKCMER) (EPSRSC grant P/R007632/1). The authors would also like to acknowledge the use of the University of Oxford Advanced Research Computing (ARC) facility in carrying out the numerical work.

\section{Appendix A.}

The reconstruction of the nonlinear force and moment relies on the Stokes-like model of the harmonic components. We first write the linear component as

$$
F^{(1)}=\mathcal{F}^{(1)} f_{1}
$$


where $f_{1}$ carries the group structure and phase information for the linear loading, and $\mathcal{F}^{(1)}$ carries the information of the linear force amplitude. Then the total force can be estimated as

$$
\begin{aligned}
\frac{F}{\rho g R^{3}} & =\frac{\mathcal{F}^{(1)}}{\rho g R^{3}} f_{1}+S_{F F 2}\left(\frac{\mathcal{F}^{(1)}}{\rho g R^{3}}\right)^{2} f_{2}+S_{F F 3}\left(\frac{\mathcal{F}^{(1)}}{\rho g R^{3}}\right)^{3} f_{3} \\
& +S_{F F 4}\left(\frac{\mathcal{F}^{(1)}}{\rho g R^{3}}\right)^{4} f_{4}+S_{F F 5}\left(\frac{\mathcal{F}^{(1)}}{\rho g R^{3}}\right)^{5} f_{5}
\end{aligned}
$$

where

$$
f_{n}=\alpha_{F F n} f_{\alpha n}+\beta_{F F n} f_{\beta n}, \quad n=2,3,4,5
$$

with

$$
\begin{aligned}
& f_{\alpha 2}=f_{1}^{2}-f_{1 H}^{2} \\
& f_{\beta 2}=2 f_{1} f_{1 H} \\
& f_{\alpha 3}=f_{1}\left(f_{1}^{2}-2 f_{1 H}^{2}\right) \\
& f_{\beta 3}=f_{1 H}\left(3 f_{1}^{2}-f_{1 H}^{2}\right) \\
& f_{\alpha 4}=\left(f_{1}^{2}-f_{1 H}^{2}\right)^{2}-\left(2 f_{1} f_{1 H}\right)^{2} \\
& f_{\beta 4}=4 f_{1} f_{1 H}\left(f_{1}^{2}-f_{1 H}^{2}\right) \\
& f_{\alpha 5}=\left[\left(f_{1}^{2}-f_{1 H}^{2}\right)^{2}-\left(2 f_{1} f_{1 H}\right)^{2}\right] f_{1}-4 f_{1 H}^{2} f_{1}\left(f_{1}^{2}-f_{1 H}^{2}\right) \\
& f_{\beta 5}=\left[\left(f_{1}^{2}-f_{1 H}^{2}\right)^{2}-\left(2 f_{1} f_{1 H}\right)^{2}\right] f_{1 H}+4 f_{1}^{2} f_{1 H}\left(f_{1}^{2}-f_{1 H}^{2}\right)
\end{aligned}
$$

At each higher harmonic, $S_{F F n}$ carries the non-dimensional force coefficient, and the phase coefficients $\alpha_{F F n}$ and $\beta_{F F n}$ are approximated by

$$
\alpha_{F F n}=\frac{\int f_{n} f_{\alpha n} \mathrm{~d} t}{\int f_{\alpha n}^{2} \mathrm{~d} t}, \quad \beta_{F F n}=\frac{\int f_{n} f_{\beta n} \mathrm{~d} t}{\int f_{\beta n}^{2} \mathrm{~d} t} .
$$

The phase of the harmonic force relative to the linear force component is then defined as $\phi_{n}=$ $\arctan \left(\beta_{F F n} / \alpha_{F F n}\right)$.

The moment reconstruction model is similar to the force as

$$
\begin{aligned}
\frac{M}{\rho g R^{3} h} & =\frac{\mathcal{M}^{(1)}}{\rho g R^{3} h} m_{1}+S_{M M 2}\left(\frac{\mathcal{M}^{(1)}}{\rho g R^{3} h}\right)^{2} m_{2}+S_{M M 3}\left(\frac{\mathcal{M}^{(1)}}{\rho g R^{3} h}\right)^{3} m_{3} \\
& +S_{M M 4}\left(\frac{\mathcal{M}^{(1)}}{\rho g R^{3} h}\right)^{4} m_{4}+S_{M M 5}\left(\frac{\mathcal{M}^{(1)}}{\rho g R^{3} h}\right)^{5} m_{5}
\end{aligned}
$$


with $S_{M M n}$ as the $n$th harmonic moment coefficient and $m_{n}$ carrying the group structure and phase information.

Finally, given the effective moment $\operatorname{arm} h_{n}$ at each harmonic, one would be able to approximate the moment from the force by

$$
\begin{aligned}
\frac{M}{\rho g R^{3} h} & =\frac{h_{1}}{h} \frac{\mathcal{F}^{(1)}}{\rho g R^{3}} f_{1}+S_{F F 2} \frac{h_{2}}{h}\left(\frac{\mathcal{F}^{(1)}}{\rho g R^{3}}\right)^{2} f_{2}+S_{F F 3} \frac{h_{3}}{h}\left(\frac{\mathcal{F}^{(1)}}{\rho g R^{3}}\right)^{3} f_{3} \\
& +S_{F F 4} \frac{h_{4}}{h}\left(\frac{\mathcal{F}^{(1)}}{\rho g R^{3}}\right)^{4} f_{4}+S_{F F 5} \frac{h_{5}}{h}\left(\frac{\mathcal{F}^{(1)}}{\rho g R^{3}}\right)^{5} f_{5} .
\end{aligned}
$$

Chaplin, J. R., Rainey, R. C. T., Yemm, R. W., 1997. Ringing of a vertical cylinder in waves. Journal of Fluid Mechanics 350, 119-147.

Chau, F. P., Eatock Taylor, R., 1992. Second-order wave diffraction by a vertical cylinder. Journal of Fluid Mechanics 240, 571-599. 
Chen, L., Stagonas, D., Santo, H., Buldakov, E., Simons, R., Taylor, P., Zang, J., 2019. Numerical modelling of interactions of waves and sheared currents with a surface piercing vertical cylinder. Coastal Engineering 145, 65-83.

Chen, L. F., Zang, J., Taylor, P. H., Sun, L., Morgan, G. C. J., Grice, J., Orszaghova, J., Tello Ruiz, M., 2018. An experimental decomposition of nonlinear forces on a surface-piercing column: Stokes-type expansions of the force harmonics. Journal of Fluid Mechanics 848, 42-77.

Dalzell, J., 1999. A note on finite depth second-order wave-wave interactions. Applied Ocean Research 21 (3), 105-111.

Faltinsen, O. M., Newman, J. N., Vinje, T., 1995. Nonlinear wave loads on a slender vertical cylinder. Journal of Fluid Mechanics 289, 179-198.

Feng, X., Willden, R. H., Zhou, B., Adcock, T. A. A., 2019. Numerical analysis of nonlinear wave loads on an offshore wind turbine monopile. In: Proceedings of the ASME 2019 38th International Conference on Ocean, Offshore and Arctic Engineering, Glasgow, UK.

Fitzgerald, C. J., Taylor, P. H., Eatock Taylor, R., Grice, J., Zang, J., 2014. Phase manipulation and the harmonic components of ringing forces on a surface-piercing column. Proceedings of the Royal Society A: Mathematical, Physical and Engineering Sciences 470 (2168).

Grue, J., Huseby, M., 2002. Higher-harmonic wave forces and ringing of vertical cylinders. Applied Ocean Research 24 (4), 203-214.

Huang, J. B., Eatock Taylor, R., 1996. Semi-analytical solution for second-order wave diffraction by a truncated circular cylinder in monochromatic waves. Journal of Fluid Mechanics 319, $171-196$.

Huseby, M., Grue, J., 2000. An experimental investigation of higher-harmonic wave forces on a vertical cylinder. Journal of Fluid Mechanics 414, 75-103.

Kallehave, D., Byrne, B. W., Thilsted, C. L., Mikkelsen, K. K., 2015. Optimization of monopiles for offshore wind turbines. Phil. Trans. R. Soc. A 373 (2035), 20140100.

Kim, M. H., Yue, D. K. P., 1989. The complete second-order diffraction solution for an axisymmetric body Part 1. monochromatic incident waves. Journal of Fluid Mechanics 200, 235-264. 
Kristiansen, T., Faltinsen, O. M., 2017. Higher harmonic wave loads on a vertical cylinder in finite water depth. Journal of Fluid Mechanics 833, 773-805.

Krokstad, J. R., Stansberg, T. C., June 1995. Ringing load models verified against experiments. In: Proceedings of 14th International Conference on Offshore Mechanics and Arctic Engineering, Copenhagen, Denmark. Vol. 1. NY: ASME, pp. 223-234.

Lo, E., Mei, C. C., 1985. A numerical study of water-wave modulation based on a higher-order nonlinear Schrödinger equation. Journal of Fluid Mechanics 150, 395-416.

Ma, Y., Dong, G., Perlin, M., Liu, S., Zang, J., Sun, Y., 2009. Higher-harmonic focused-wave forces on a vertical cylinder. Ocean engineering 36 (8), 595-604.

Malenica, S., Molin, B., 1994. Third-order triple frequency wave forces on fixed vertical cylinders. In: Proceedings of 9th International Workshop on Water Waves and Floating Bodies, Kyushu, Japan. Institute for Applied Mechanics, Kyushu University.

Malenica, S., Molin, B., 1995. Third-harmonic wave diffraction by a vertical cylinder. Journal of Fluid Mechanics 302, 203-229.

Molin, B., Remy, F., Kimmoun, O., Jamois, E., 2005. The role of tertiary wave interactions in wave-body problems. Journal of Fluid Mechanics 528, 323-354.

Newman, J. N., 1996. The second-order wave force on a vertical cylinder. Journal of Fluid Mechanics $320,417-443$.

Riise, B. H., Grue, J., Jensen, A., Johannessen, T. B., 2018a. High frequency resonant response of a monopile in irregular deep water waves. Journal of Fluid Mechanics 853, 564-586.

Riise, B. H., Grue, J., Jensen, A., Johannessen, T. B., 2018b. A note on the secondary load cycle for a monopile in irregular deep water waves. Journal of Fluid Mechanics 849, R1.

Santo, H., Taylor, P. H., Day, A. H., Nixon, E., Choo, Y. S., 2018. Blockage and relative velocity morison forces on a dynamically-responding jacket in large waves and current. Journal of Fluids and Structures 81, 161-178.

Sharma, J. N., Dean, R. G., 1981. Second-order directional seas and associated wave forces. Society of Petroleum Engineers Journal 21 (01), 129-140. 
Stansberg, C. T., 1997. Comparing ringing loads from experiments with cylinders of different diameters - An empirical study. In: Proc. 8th Conf. on the Behaviour of Offshore Structures (BOSS'97). Vol. 2. Pergamon/Elsevier, pp. 95-112.

Teng, B., Kato, S., 2002. Third order wave force on axisymmetric bodies. Ocean Engineering $29(7), 815-843$.

Tromans, P. S., Anaturk, A. R., Hagemeijer, P., 1991. A new model for the kinematics of large ocean waves-application as a design wave. In: The First International Offshore and Polar Engineering Conference. International Society of Offshore and Polar Engineers.

Zhao, W., Wolgamot, H. A., Taylor, P. H., Eatock Taylor, R., 2017. Gap resonance and higher harmonics driven by focused transient wave groups. Journal of Fluid Mechanics 812, 905-939. 
- Higher harmonics of wave loading on offshore wind turbine monopiles is investigated using experimental and numerical methods

- Non-linearity in forces and moments can be captured using a "Stokes-type" approach

- Third order forces and moments so significantly more variation than second, fourth and fifth harmonics

- The approach can be used to quickly generate timeseries of wave loading with a good representation of the higher harmonics for geotechnical and structural calculations 


\section{Declaration of interests}

$\bigotimes$ The authors declare that they have no known competing financial interests or personal relationships that could have appeared to influence the work reported in this paper.

$\square$ The authors declare the following financial interests/personal relationships which may be considered as potential competing interests: 